\title{
Science and Curation Considerations for the Design of a Mars Sample Return (MSR) Sample Receiving Facility (SRF)
}

Brandi L. Carrier, ${ }^{1}$ David W. Beaty, ${ }^{1}$ Aurore Hutzler, ${ }^{2}$ Alvin L. Smith, ${ }^{1}$ Gerhard Kminek, ${ }^{2}$ Michael A. Meyer, ${ }^{3}$ Timothy Haltigin, ${ }^{4}$ Lindsay E. Hays, ${ }^{3}$ Carl B. Agee, ${ }^{5}$ Henner Busemann, ${ }^{6}$ Barbara Cavalazzi, ${ }^{7}$ Charles S. Cockell, ${ }^{8}$ Vinciane Debaille, ${ }^{9}$ Daniel P. Glavin, ${ }^{10}$ Monica M. Grady, ${ }^{11}$ Ernst Hauber, ${ }^{12}$ Bernard Marty, ${ }^{13}$ Francis M. McCubbin, ${ }^{14}$ Lisa M. Pratt, ${ }^{15}$ Aaron B. Regberg, ${ }^{14}$ Caroline L. Smith,,${ }^{16,17}$ Roger E. Summons, ${ }^{18}$ Timothy D. Swindle, ${ }^{19}$ Kimberly T. Tait, ${ }^{20}$ Nicholas J. Tosca, ${ }^{21}$ Arya Udry, ${ }^{22}$ Tomohiro Usui ${ }^{23}$ Michael A. Velbel, ${ }^{24,25}$ Meenakshi Wadhwa, ${ }^{1,26}$ Frances Westall, ${ }^{27}$ and Maria-Paz Zorzano ${ }^{28,29}$

${ }^{1}$ Jet Propulsion Laboratory, California Institute of Technology, Pasadena, California, USA.

${ }^{2}$ European Space Agency, Noordwijk, The Netherlands.

${ }^{3}$ NASA Headquarters, Mars Sample Return Program, Washington, DC, USA.

${ }_{5}^{4}$ Canadian Space Agency, Saint Hubert, Quebec, Canada.

${ }^{5}$ University of New Mexico, Institute of Meteoritics, Albuquerque, New Mexico, USA.

${ }^{6}$ ETH Zürich, Institute of Geochemistry and Petrology, Zürich, Switzerland.

${ }^{7}$ Università di Bologna, Dipartimento di Scienze Biologiche, Geologiche e Ambientali, Bologna, Italy.

${ }^{8}$ University of Edinburgh, Centre for Astrobiology, School of Physics and Astronomy, Edinburgh, UK.

${ }^{9}$ Université Libre de Bruxelles, Bruxelles, Belgium.

${ }^{10}$ NASA Goddard Space Flight Center, Solar System Exploration Division, Greenbelt, Maryland, USA.

${ }^{11}$ The Open University, Milton Keynes, UK.

${ }^{12}$ German Aerospace Center (DLR), Institute of Planetary Research, Berlin, Germany.

${ }^{13}$ Université de Lorraine, CNRS, CRPG, Nancy, France.

${ }_{15}^{14}$ NASA Johnson Space Center, Astromaterials Research and Exploration Science Division, Houston, Texas, USA.

${ }^{15}$ Indiana University Bloomington, Earth and Atmospheric Sciences, Bloomington, Indiana, USA.

${ }^{16}$ Natural History Museum, Department of Earth Sciences, London, UK.

${ }^{17}$ University of Glasgow, School of Geographical and Earth Sciences, Glasgow, UK.

${ }^{18}$ Massachusetts Institute of Technology, Earth, Atmospheric and Planetary Sciences, Cambridge, Massachusetts, USA.

${ }^{19}$ University of Arizona, Lunar and Planetary Laboratory, Tucson, Arizona, USA.

${ }^{20}$ Royal Ontario Museum, Department of Natural History, Toronto, Ontario, Canada.

${ }_{21}^{21}$ University of Cambridge, Department of Earth Sciences, Cambridge, UK.

${ }^{22}$ University of Nevada Las Vegas, Las Vegas, Nevada, USA.

${ }^{23}$ Japan Aerospace Exploration Agency (JAXA), Institute of Space and Astronautical Science (ISAS), Chofu, Tokyo, Japan.

${ }^{24}$ Michigan State University, Earth and Environmental Sciences, East Lansing, Michigan, USA.

${ }^{25}$ Smithsonian Institution, Department of Mineral Sciences, National Museum of Natural History, Washington, DC, USA.

${ }^{26}$ Arizona State University, Tempe, Arizona, USA.

${ }^{27}$ Centre National de la Recherche Scientifique (CNRS), Centre de Biophysique Moléculaire, Orléans, France.

${ }^{28}$ Centro de Astrobiologia (CSIC-INTA), Torrejon de Ardoz, Spain.

${ }^{29}$ University of Aberdeen, Department of Planetary Sciences, School of Geosciences, King's College, Aberdeen, UK.

This paper was written by the MSR Science Planning Group 2 (MSPG2) working under a Terms of Reference from NASA and ESA.

(C) Brandi L. Carrier et al., 2021; Published by Mary Ann Liebert, Inc. This Open Access article is distributed under the terms of the Creative Commons License (http://creativecommons.org/licenses/by/4.0), which permits unrestricted use, distribution, and reproduction in any medium, provided the original work is properly cited. 


\section{Table of Contents}

Abstract

Executive Summary

Summary of Findings

1. Introduction

1.1 Overview

1.2 Previous work and context of SRF planning

1.2.1 1990s

1.2.2 '03/'05 MSR and the introduction to MRSH, 1999-2004

1.2.3 Inter-agency planning, 2005-2017

1.2.4 MSR Science Planning Group (MSPG, MSPG2), 2018-2021

1.3 SRF objectives and assumptions

1.3.1 SRF objectives

1.3.2 Assumptions

1.4 Technical notes

2. Core Science and Curation Functionalities

2.1 Preserving the scientific value of the samples

2.1.1 Environmental conditions

2.1.2 Contamination control

2.2 Analytical capabilities

2.2.1 Curation-initial sample characterization

2.2.2 Time-sensitive measurements

2.2.3 Sterilization-sensitive measurements

2.2.4 Sample Safety Assessment Protocol

2.2.5 Sample processing, preparation, and storage

2.2.6 Considerations for gas retrieval and analysis

2.2.7 Planning for Analytical instrumentation in the SRF

3. Other SRF Capabilities

3.1 Overall biocontainment building engineering and infrastructure

3.1.1 Capabilities requirements

3.2 Spacecraft receiving and de-integration

3.3 Planetary Protection: Sterilization

3.4 Sample prep/packaging/shipping

4. Discussion

5. Recommendations for Future Work

Acknowledgments

Disclosure Statement

Funding Information

References

Acronyms Used

\section{Abstract}

The most important single element of the "ground system" portion of a Mars Sample Return (MSR) Campaign is a facility referred to as the Sample Receiving Facility (SRF), which would need to be designed and equipped to receive the returned spacecraft, extract and open the sealed sample container, extract the samples from the sample tubes, and implement a set of evaluations and analyses of the samples.

One of the main findings of the first MSR Sample Planning Group (MSPG, 2019a) states that "The scientific community, for reasons of scientific quality, cost, and timeliness, strongly prefers that as many sample-related investigations as possible be performed in PI-led laboratories outside containment." There are many scientific and technical reasons for this preference, including the ability to utilize advanced and customized instrumentation that may be difficult to reproduce inside in a biocontained facility, and the ability to allow multiple science investigators in different labs to perform similar or complementary analyses to confirm the reproducibility and accuracy of results. It is also reasonable to assume that there will be a desire for the SRF to be as efficient and economical as possible, while still enabling the objectives of MSR to be achieved.

For these reasons, MSPG concluded, and MSPG2 agrees, that the SRF should be designed to accommodate only those analytical activities that could not reasonably be done in outside laboratories because they are time- 
or sterilization-sensitive, are necessary for the Sample Safety Assessment Protocol (SSAP), or are necessary parts of the initial sample characterization process that would allow subsamples to be effectively allocated for investigation. All of this must be accommodated in an SRF, while preserving the scientific value of the samples through maintenance of strict environmental and contamination control standards.

\section{Executive Summary}

The most important single element of the "ground system" portion of a Mars Sample Return (MSR) Campaign is a facility referred to as the Sample Receiving Facility (SRF), which would need to be designed and equipped to enable receipt of the returned spacecraft, extraction and opening of the sealed sample container, extraction of the samples from the sample tubes, and a set of evaluations and analyses of the samples-all under strict protocols of biocontainment and contamination control. Some of the important constraints in the areas of cost and required performance have not yet been set by the necessary governmental sponsors, but it is reasonable to assume there will be a desire for the SRF to be as efficient and economical as is possible, while still enabling the objectives of MSR science to be achieved. Additionally, one of the main findings of MSR Sample Planning Group (MSPG, 2019a) states "The scientific community, for reasons of scientific quality, cost, and timeliness, strongly prefers that as many sample-related investigations as possible be performed in PI-led laboratories outside containment." There are many scientific and technical reasons for this preference, including the ability to utilize advanced and customized instrumentation that may be difficult to reproduce inside a biocontained facility. Another benefit is the ability to enable similar or complementary analyses by multiple science investigators in different laboratories, which would confirm the reproducibility and accuracy of results.

For these reasons, the MSPG concluded-and the MSR Science Planning Group Phase 2 (MSPG2) agrees-that the SRF should be designed to accommodate only those analytical activities inside biocontainment that could not reasonably be done in outside laboratories because such activities are time-sensitive, sterilization-sensitive, required by the Sample Safety Assessment Protocol (SSAP), or are necessary parts of the initial sample characterization process that would allow subsamples to be effectively allocated for investigation. All activities within the SRF must be done while preserving the scientific value of the samples through maintenance of strict environmental and contamination control standards.

The SRF would need to provide a unique environment that consists of both Biosafety Level 4 (BSL-4) equivalent containment and a very high level of contamination control. The SRF would also need to accommodate the following activities:

(1) Receipt of the returned spacecraft, presumably in a sealed shipping container

(2) De-integration (i.e., disassembly) and assessment of the returned system, beginning with the spacecraft exterior and ending with accessing and isolating all Mars material (gas, dust, regolith, and rock)

(3) Initial sample characterization, leading to development of a sample catalog sufficient to support sample allocation (see Tait et al., 2022)

(4) Science investigations necessary to complete the SSAP (see Kminek et al., 2021)
(5) Certain science investigations that are both time- and sterilization-sensitive (see Tosca et al., 2022; Velbel et al., 2022)

(6) A managed transition to post-SRF activities that would include analysis of samples (either sterilized or not) outside biocontainment and the transfer of some or all samples to one or more uncontained curation facilities

The MSPG2 has produced a compilation of potential design requirements for the SRF, based on the list of activities noted above, that can be used in cost and schedule planning. The text of this report is meant to serve as an overview and explanation of these proposed SRF Design Requirements that have been compiled by the MSPG2 SRF Requirements Focus Group (Supplement 1).

\section{Summary of Findings}

FINDING SRF-1: The quality of the science that can be achieved with the MSR samples will be negatively impacted if they are not protected from contamination and inappropriate environmental conditions. A significant amount of SRF infrastructure would therefore be necessary to maintain and monitor appropriate levels of cleanliness, contamination control, and environmental conditions.

FINDING SRF-2: Although most MSR sample investigations would take place outside of the SRF, the SRF needs to include significant laboratory capabilities with advanced instruments and associated sample preparation systems to enable the MSR science objectives to be successfully achieved.

FINDING SRF-3: Preliminary studies of different operational scenarios should be started as soon as possible to enable analysis of the trade-offs between the cost and size of the SRF and the amount of time needed to prepare the samples for allocation and analysis.

FINDING SRF-4: The ability to add additional analytical capabilities within biocontainment should be preserved to address the contingency scenario in which unsterilized material is not cleared to be analyzed outside of biocontainment. If potential evidence of martian life were to be detected in the samples, for example, it would be a high priority to conduct further investigations related to any putative lifeforms, as well as to enable other sterilization-sensitive science investigations to be conducted in biocontainment.

\section{Introduction}

\subsection{Overview}

Tn 2018, NASA and the European Space Agency (ESA) signed a joint Statement of Intent to continue defining respective roles and responsibilities in the flight missions required to realize MSR. In October 2020, NASA and ESA formalized this partnership with the signature of a Memorandum of Understanding for the MSR flight missions. The returned samples would be carefully managed and made available to the international community for scientific 
investigation. The current baseline architecture for the MSR Campaign consists of the following: (i) NASA's Mars 2020 Perseverance rover (currently in operation on Mars), which will collect and encapsulate samples, (ii) two joint ESA-NASA flight missions that are intended to retrieve the samples from Mars and return them to Earth (both in development as of this writing), and (iii) the ground-based systems needed to receive and process the samples once they arrive on Earth. The MSR flight missions represent one of the most complex undertakings that NASA and ESA have ever attempted and are hereafter collectively referred to as the "MSR Program.",

The planning for what would happen to the samples when they arrive on Earth is comparably complex, although for different reasons. The most important single element of the "ground system" is a facility referred to as the Sample Receiving Facility (SRF), where the returned spacecraft would be opened and the sealed sample container removed to access the samples. The individual sample tubes would be examined before being opened, followed by extraction and evaluation of the samples through a series of analytical procedures. Each of the steps would be carried out under strict protocols of biocontainment and environmental and contamination control.

In response to the Terms of Reference (ToR) of the MSPG2 activity chartered by NASA and ESA in March 2020 (MSPG2 ToR, Meyer et al., 2022), this report addresses Deliverable \#3: "Develop approaches and a working list of high-level requirements for the SRF that can be used in cost estimation and budgeting. The requirements specifically need to represent the needs and interests of each of science, curation, and planetary protection. All proposed requirements need a justification statement." This report, which represents our response to this request in the form of a narrative, explains assumptions, strategies, relationships, etc. and provides a table of proposed requirements (Supplement 1). Note that as we built the list of proposed requirements we chose to limit our scope to SRF design requirements, which would affect cost and schedule, rather than operating requirements. The latter will need further work by a successor planning group.

Our report is a direct extension of three companion reports prepared concurrently by MSPG2 over the past nine months, which analyze three technical areas that would need to be managed as part the work in the SRF as follows: sample curation, time-sensitive science, and the effects of sample sterilization. It is the authors' intent that this report compiles all of the requirements mentioned in the other documents, as well as some derived from elsewhere.

\subsection{Previous work and context of SRF planning}

1.2.1. 1990s. A number of reports from studies and workshops during the 1990s established the context for SRF design and implementation. IMEWG (the International Mars Exploration Working Group) was established in 1993 to ensure that planning for Mars exploration (including sample return) was coordinated across different space agencies, encouraging inter-agency co-operation. In 1995, NASA asked the National Research Council (NRC) to conduct a study pertaining to sample return that addressed key issues associated with the potential risks to Earth from samples returned from Solar System bodies such as Mars. The NRC panel focused principally on Mars, producing a report (National Research Council, 1997) that became a cornerstone for much of the planetary protection-related work of the MSR '03-'05 Project. In addition, the NASA Mars Sample Handling and Requirements Panel (MSHARP) made recommendations (Carr et al., 1999) with regard to certification of returned samples as non-hazardous and considerations associated with sample receiving, curation, and distribution. MSHARP recommended that the samples be treated as hazardous until proven otherwise, which is consistent with NRC (1997).

1.2.2. '03/'05 MSR and the introduction to MRSH, 19992004. The first in-depth discussion of facility planning for an MSR-related Sample Receiving Facility was carried out in connection with planning for the MSR '03-'05 Project. This version of MSR advanced as far as Preliminary Design Review (PDR) before it was cancelled in 2000. Regarding SRF planning, a workshop was held in February 1999 at Caltech called the Mars Sample Handling, Distribution, and Analysis Workshop (D. McCleese and M. Drake, Chairs) that introduced the term "Mars Receiving Facility." This facility concept was renamed later that year to the more generic "Sample Receiving Facility" (or SRF) by NASA's newly formed Mars Returned Sample Handling (MRSH) team because of the possibility that such a facility might be used in the future for samples originating from planetary objects other than Mars. Other synonymous terms used at that time were "Sample Handling Facility" (SHF) and "Mars Handling Facility" (MHF), but these were discarded because of unfavorable connotations around the word "Handling."

Concurrent with this work was a very important set of activities led by John Rummel and several colleagues to develop a first draft of the Planetary Protection test protocol. This led to the publication of several workshop reports, and a summary roll-up document (Rummel et al., 2002). This activity touched on the measurements needed in the SRF to assess sample safety as well as general concepts for the organization and management of the SRF.

At the end of the '03/'05 Project, NASA recognized that, if an MSR project were restarted in the future, it would have to include realistic planning parameters for the SRF, including cost, schedule, and size. Therefore, planning activity for the SRF continued through 2004, several years beyond the cancellation of the flight mission. The MRSH team (key members included David Beaty, David Lindstrom, Dimitri Papanastassiou, and Carlton Allen) made some valuable advancements in understanding approximate cost, schedule, technology needs, and relationship to the flight program. A major aspect of this planning was a set of three independently completed industry studies that were carried out in 20032004, the engineering of which was led by Richard Mattingly, Jim Campbell, and Frank Jordan. These studies formed the basis for a much clearer understanding of the possible requirements, design, cost, timeline, and operational considerations for an MSR-related SRF, and a summary of these studies was published by Beaty et al. (2009).

Because the earlier reports provided advice at a fairly general level, the NRC followed up with a study of the criteria for release of samples from biocontainment in an SRF (National Research Council, 2002). Of relevance to the present study, the report recommended that only the most basic operations be conducted inside the facility, and it should be designed to the smallest and simplest possible scale consistent with its dual roles as a biological 
containment and cleanroom facility, with detailed protocols and procedures for handling and testing Mars samples.

1.2.3. Inter-agency planning, 2005-2017. The international Mars Architecture for the Return of Samples Working Group (iMARS WG), which was active in 2007-2008 under the sponsorship of the International Mars Exploration Working Group (IMEWG), represented a concentrated effort to internationalize MSR (Beaty et al., 2008). Although much of their activity focused on the flight systems, one of their working groups evaluated management of the samples after they returned to Earth, and in a general sense, the functionality of the SRF. They proposed that the samples be managed by an international institute, for which they applied the conceptual acronym IMSI (International MSR Science Institute). CERN (The European Organization for Nuclear Research) and STSI (Space Telescope Science Institute) were identified as potentially analogous institutes. iMARS Phase 2 (iMARS-2) was then subsequently tasked with evaluating the IMSI concept, and they proposed some initial concepts for the management of MSR science, including the SRF (Haltigin et al., 2018).

In 2009, the Visions \& Voyages Planetary Science Decadal Survey stated that the highest priority flagship mission for 2013-2022 should be a Mars sample-collecting rover that would represent the first step in a joint NASA-ESA MSR Campaign (National Research Council, 2011). This led to the development, launch, and subsequent successful landing of the Mars 2020 Perseverance rover in 2021. The MSR End-to-End International Science Analysis Group (E2EiSAG) was formed to ensure that the sample collection by Mars 2020 was properly planned in the larger context of MSR science. This group consolidated and prioritized the overarching science aims and objectives for the MSR campaign, and made recommendations for the types, number, and character of samples needed to achieve the MSR science objectives (McLennan et al.,2012). The recommendations of E2E-iSAG were used to inform the design of the Mars 2020 rover and its sampling system, as well as the selection of its instrument suite. The international MSR Objectives and Samples Team (iMOST) was chartered in 2017 to refine the science objectives for MSR and determine what types of measurements would need to be made to achieve those objectives (Beaty et al., 2019). These reports served as the starting point for many of the discussions of MSPG and MSPG2.

1.2.4. MSR Science Planning Group (MSPG, MSPG2), 2018-2021. In 2018-19, the ESA- and NASA-chartered MSR Science Planning Group (MSPG) developed an updated set of science-related strategies to guide SRF planning (MSPG, 2019a, b). This planning incorporated all of the prior planning work listed above and, in particular, made use of some of the concepts developed by iMARS-2. These strategies better reflected current NASA, ESA, and community attitudes and priorities relating to the SRF.

\subsection{SRF objectives and assumptions}

The proposed design requirements for the SRF (Supplement 1) have been developed based on the following lists of objectives and assumptions. To maximize the scientific output of the samples and minimize the cost and size of the SRF, this list and the resulting proposed requirements were written with the intent that as many analyses as possible should be conducted outside the SRF, either on sterilized samples or after the samples have been determined to be safe for release.

1.3.1. SRF objectives. The SRF would need to provide a unique environment that would consist of both BSL-4 equivalent containment (Section 3.1) and a very high level of contamination control (Section 2.1), and it would need to accommodate the following activities:

(1) Receipt of the returned spacecraft, presumably in a sealed shipping container (Section 3.2)

(2) De-integration (i.e., disassembly) and assessment of the returned system, beginning from the spacecraft exterior and ending with accessing and isolating all Mars material (gas, dust, regolith, and rock) (Section 3.2)

(3) Initial sample characterization, leading to development of a sample catalog sufficient to support sample allocation (see Tait et al., 2022; Section 2.2.1 of this report)

(4) Science investigations necessary to complete the Sample Safety Assessment Protocol (see Kminek et al., 2021; Section 2.2.4 of this report)

(5) Certain science investigations that are both time- and sterilization-sensitive (see Tosca et al., 2022 and Velbel et al., 2022; Sections 2.2.2 and 2.2.3 of this report)

(6) A managed transition to post-SRF activities, including analysis of samples (either sterilized or not) outside biocontainment, and the transfer of some or all samples to one or more uncontained curation facilities (Sections 2.2.5, 3.3, 3.4)

\subsubsection{Assumptions}

(1) The landing site of the MSR Earth Entry System (EES) would be in the U.S., and one SRF would also be located in the U.S. (MSPG2 ToR, Meyer et al., 2022). The possibility of a second biocontained sample facility in Europe exists but is not addressed here, because the potential existence of a second biocontained facility is not expected to impact the necessary functionalities for the primary SRF.

(2) A primary purpose of the SRF would be to preserve the scientific value of the samples, which will necessitate requirements related to environmental and contamination control.

(3) The SRF would be a tailored facility-sufficiently capable to achieve the objectives stated above. All other measurements should be planned to take place outside biocontainment on samples that have been sterilized or have been determined not to contain biohazardous material.

(4) One of the primary MSPG findings states that "The scientific community, for reasons of scientific quality, cost, and timeliness, strongly prefers that as many sample-related investigations as possible be performed in PI-led laboratories outside containment." A direct implication is that scientific investigations that can tolerate sterilization should be done outside biocontainment (MSPG, 2019a).

(5) In addition to planetary protection-, science-, and curation-driven requirements, the SRF would also have requirements related to building engineering, infrastructure, physical security, and other traditional facilities-related requirements. 


\subsection{Technical notes}

Capabilities related to curation (including initial sample characterization), sterilization-sensitive measurements, and time-sensitive measurements, all of which are necessary to complete the Sample Safety Assessment Protocol (SSAP), are outlined in Section 2. The reports on these activities by their respective MSPG2 Focus Groups provide a higher level of detail with regard to their findings and reasoning (Tait et al., 2022; Tosca et al., 2022; Velbel et al., 2022).

Section 3 is a compilation of objectives and functionalities for the SRF that have been formulated over the past two decades, which include the need for BSL-4 equivalent containment and to meet all requirements related to planetary protection, overall building engineering functions, and spacecraft receiving and de-integration capabilities. We note the following about the approach taken to develop the proposed SRF Design Requirements table given in Supplement 1:

(1) We limited our analysis to requirements that relate to facility design and not to its operation. Analysis of operational requirements, including procedures, personnel functionalities and relationships, training, etc., should be the focus of a future report.

(2) As was noted by Mattingly et al. (2020), there are several ways for facilities of this general type to be configured, and we would like to allow and encourage the blending of best practices for this unique facility. For this reason, we have defined the proposed requirements in terms of necessary capabilities and are not advocating for specific implementations. An exception, however, is that to convey a better understanding of the instruments that may need to be accommodated in the SRF, we have named what we are calling "reference instruments.", Our definition of this term, and how we have used it, is treated below in the introduction to Section 2.2.

(3) We have used a hierarchical requirements structure that begins with Level 1 (L1) at the most general. We consider our analysis of the L1 requirements to be reasonably complete, but for L2 and L3 we have strived to collect the requirements that might affect overall facility cost and schedule and deliberately did not strive for completeness (future follow up work is needed). Some of the requirements at L2 and L3 effectively serve as placeholder requirements, where the values will need to be filled in by a future group. For example, we know there will be a numerical value for allowable organic contamination, or rate of contamination, but the details of a contamination control and knowledge plan have been deferred to a future planning group. More details on the areas identified for future work can be found in Section 5 of this report.

More information on the format and structure of the proposed requirements list is presented in Supplement 1.

\section{Core Science and Curation Functionalities}

\subsection{Preserving the scientific value of the samples}

As expressed in Assumption \#2 (Section 1.3.2), one of the primary purposes of the SRF must be to preserve the scientific value of the returned samples. The quality of the science that can be achieved by analyzing the samples is highly dependent on protecting them from alteration or degradation caused by inappropriate environmental storage conditions and terrestrial contamination. The Mars 2020 Project has numerous requirements related to limiting contamination and maintaining sample quality during collection of material on Mars (Boeder and Soares, 2020). These requirements were established to ensure that study and analysis of the MSR samples would address the planned scientific objectives within the necessary constraints of mission design and cost (Beaty et al., 2014; Carrier et al., 2017; Liu et al., 2014a,b). Some of these standards were formulated for the Mars 2020 Project by the Returned Sample Science Board (RSSB), a group chartered in mid2015 by NASA to represent the sample scientific community who will study the returned samples, while others were formulated by predecessors of the RSSB (Beaty et al., 2014; RSSB 2016a,b; 2018a,b).

The set of requirements specifically aimed at preserving the scientific integrity of the samples once they have returned to Earth parallels those designed to protect sample scientific integrity while the samples are transported to Earth. Some of the requirements for samples returned to Earth differ depending on the environment in which the samples are processed (e.g., in an isolator vs. in a laboratory environment). The requirements fall into two general categories:

(1) The capability to control and provide knowledge of environmental conditions to which the MSR samples are exposed;

(2) The capability to limit sample contamination (e.g., organic, inorganic, particulate, biological) to within acceptable limits and provide knowledge and documentation of the contamination.

To meet these requirements, the SRF would need the capability to precision clean large and small pieces of equipment, and to control and monitor various environmental conditions and contamination levels in real time.

2.1.1. Environmental conditions. To limit sample alteration and degradation, samples and subsamples should be stored and processed in controlled environments. This includes temperature and the composition and humidity of the atmospheric gas in isolators or storage containers. It also requires avoidance of exposure of the samples to magnetic fields that could interfere with measurement of magnetic properties of the samples.

With regard to temperature, the intention is to operate the $\mathrm{SRF}$ and store and process samples at $15-25^{\circ} \mathrm{C}$ (Tait et al., 2022). There may be an exception for select sample splits if they are believed to contain evidence of martian biology or other properties that would warrant storage at colder temperatures (i.e., $-20^{\circ} \mathrm{C}$ ) to further limit degradation until additional analysis can be done (Tosca et al., 2022). The proposed SRF Design Requirements table also includes requirements related to maintaining high-purity inert gas atmosphere inside the sample containers and isolators and to limiting humidity in isolators, storage containers, and laboratories. The actual allowable humidity values and planning for what type of gas environment under which to store and process samples will be defined by a future planning group. 
To verify that the environmental standards are maintained, monitoring sensors and other equipment would be needed throughout areas in which samples are processed and analyzed. The full description of these monitoring systems is outside the scope of this report but should be part of the detailed facility design process.

2.1.2. Contamination control. There are multiple types of contamination that are of concern for MSR samples. These include organic, inorganic, biological, and particulate contamination, all of which would need to be strictly controlled and monitored. MSPG has proposed using the M2020 requirements (MSPG, 2019b and references in Section 2.1 of this report) for limiting contamination by viable organisms, as well as organic and inorganic contamination, as a starting point in developing contamination requirements for the SRF, but the details of these requirements will need to be refined by a future planning group. Contamination control requirements will apply at all stages subsequent to initial receipt of the spacecraft. Figure 1 shows qualitatively those phases of the workflow that are expected to have the most stringent contamination control requirements.

To maintain and monitor appropriate levels of cleanliness, the SRF must include the infrastructure and capabilities to perform precision cleaning and final cleaning of laboratory spaces, instruments, gloveboxes, and isolators, as well as smaller equipment and tools that would come into contact with the samples. This would require the capability to sterilize equipment and tools and provide ultrapure water generation infrastructure for the cleaning facilities as well as the sample preparation laboratories. The capabilities would be needed to monitor airflow as well as particulate, inorganic, organic, and microbial contamination within air filtration systems, labs, gloveboxes, and isolators. The full description of these monitoring systems is outside the scope of this report but will need to be part of the detailed facility design process.

FINDING SRF-1: The quality of the science that can be achieved with the MSR samples will be negatively impacted if they are not protected from contamination and inappropriate environmental conditions. A significant amount of SRF infrastructure would therefore be necessary to maintain and monitor appropriate levels of cleanliness, contamination control, and environmental conditions.

\subsection{Analytical capabilities}

As noted above, MSPG2 has drafted the proposed SRF Design Requirements in terms of required capabilities, leaving implementation options open rather than dictating specific instrumentation. For example, a required capability might be the necessity to determine the mineralogy of a

\section{SRF Concept of Operations}

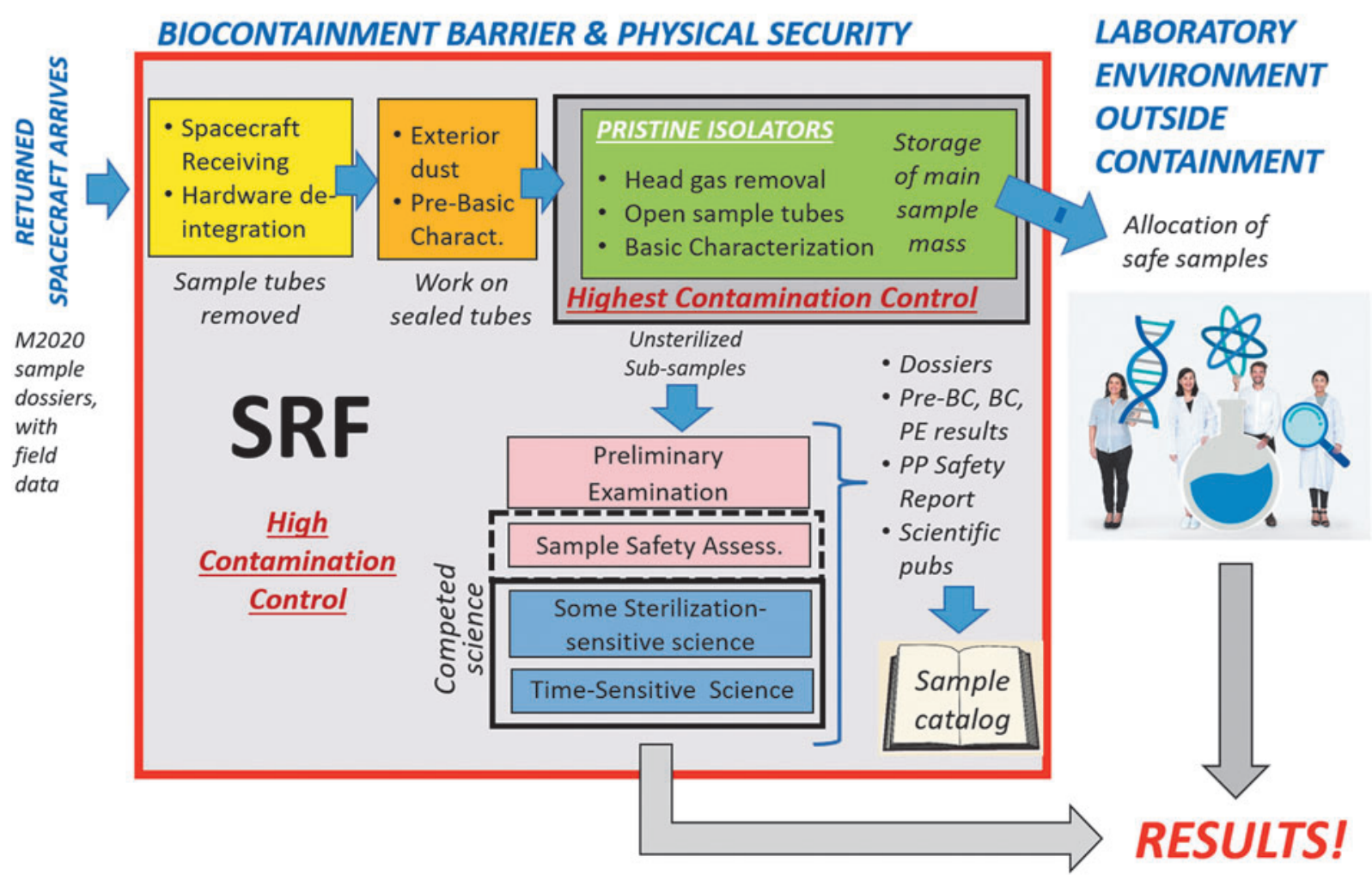

FIG. 1. Schematic workflow of SRF activities and their relative levels of contamination control. 
sample at high spatial resolution, which could be met through use of a Scanning Electron Microscope (SEM), Fourier Transform Infrared Spectroscopy (FT-IR), or Raman spectroscopy.

To convey a better understanding of the number and type of instruments that may need to be accommodated in the SRF, each of the three Focus Groups also drafted traceability matrices to correlate their specific analysis goals with the types of instruments that would be necessary and sufficient to meet those goals (Tait et al., 2022; Tosca et al., 2022; Velbel et al., 2022). Knowledge of these potential instrument types allowed MSPG2 to consider overlap between capabilities needed for different objectives and led to the naming of "reference instruments." These instruments have been compiled in Supplements 2 and 3 and are intended to be used for planning and discussion purposes only. It is expected that a future group will draft more specific instrument-level performance requirements, and these would be used to conduct a competitive procurement process.

2.2.1. Curation: Initial sample characterization. Curation is defined here as a set of activities whose main purpose is to preserve the scientific value of a sample collection, while enabling it to be used efficiently by the community seeking to realize the MSR Campaign science objectives. To fulfill this purpose, it is important to achieve a sufficient understanding of the samples and share that understanding with the community through a sample catalog. This ensures that the allocation of subsamples can be optimized for the scientific investigations for which they are intended to be used. A sample catalog would include all data and metadata linked to the samples.

The report from the Curation Focus Group (Tait et al., 2022) also describes in detail the initial sample characterization processes required to produce the sample catalog. Figure 2 shows the expected workflow for this characterization, and Table 1 summarizes the various activities considered in the work of Tait et al. (2022).
2.2.1.1. Implications for SRF capabilities. Tait et al. (2022) produced a traceability matrix that summarizes the goals of the curation activities taking place inside the SRF to complete the initial sample characterization, with corresponding measurements and reference instruments that would be sufficient to carry out those activities. The primary goals are as follows:

(1) Understand the state of the samples prior to tube opening

(2) Initiate an inventory and tracking of the samples (including grain size, density/porosity, lithology assessment)

(3) Carry out a preliminary assessment of lithology and any macroscopic forms of heterogeneity

(4) Understand sufficiently the essential attributes of each sample so that a sample catalog can be prepared and appropriate samples distributed; distribution of samples would be subject to governance guidelines

The traceability matrix and the reference instrument types, which have been incorporated into the integrated instrument list, can be viewed in Supplement 2 of this report.

To carry out the curatorial activities described in the previous section, an array of sample preparation and manipulation techniques is needed. All of these activities are expected to take place under biocontainment, while respecting the environmental and contamination requirements described in Section 2.1 and limiting cross contamination between samples. It is recommended that all of these activities should be recorded through photos, videos, and notes.

Before the sample tubes are opened, a dust removing station will be needed. It will be important to recover all dust from the outside of the sample tubes for further investigation (Grady et al., 2022). It would also be necessary to check the quality of the sample tube seals, make measurements of the sample magnetic properties, and conduct penetrative imaging at this stage.

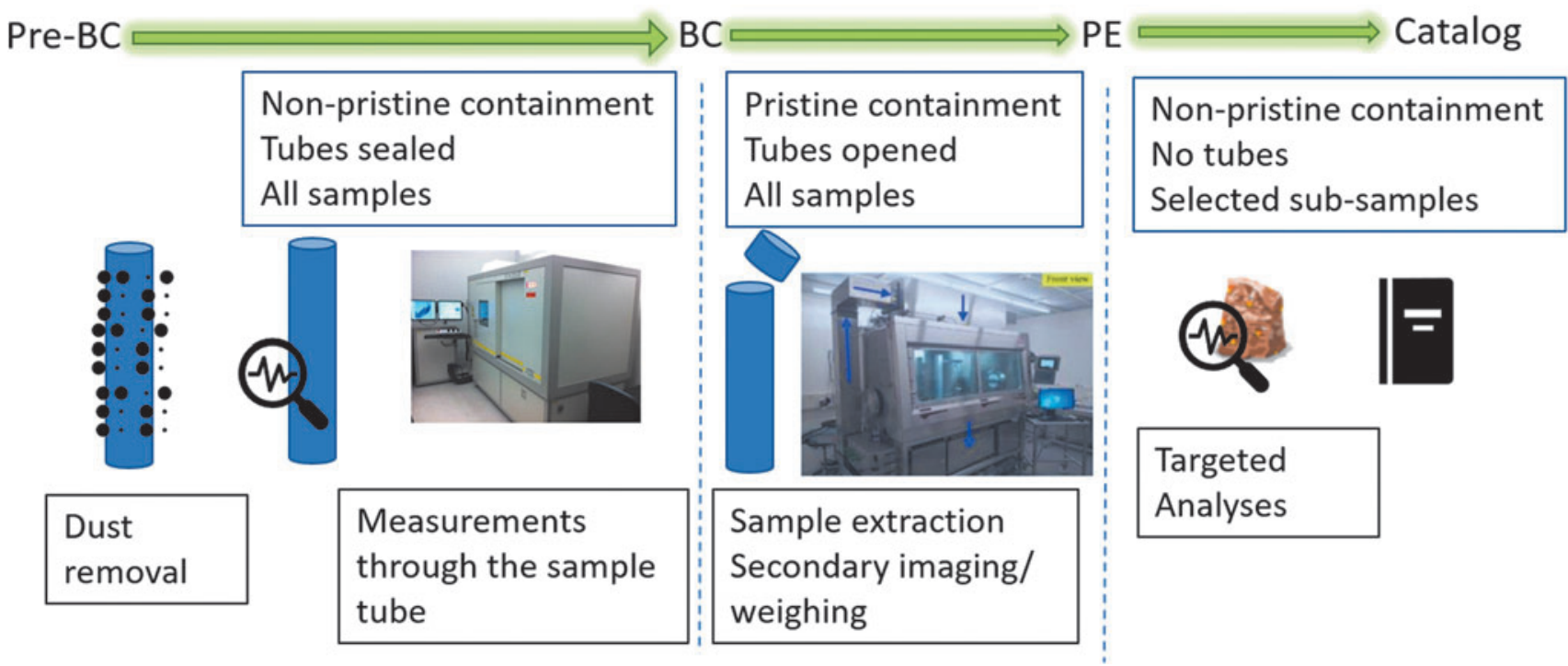

FIG. 2. Diagram of expected workflow for initial sample characterization (modified from Tait et al., 2022). 
TABle 1. The three Stages of Initial SAMPle Characterization (TAit ET AL., 2022)

\begin{tabular}{|c|c|c|c|}
\hline Stage & Environment* & Process & $\begin{array}{c}\text { Tait et al. } \\
\text { (2022) }\end{array}$ \\
\hline $\begin{array}{l}\text { Pre-Basic Characterization (Pre-BC): } \\
\text {-Measurements that would be lost } \\
\text { or compromised during tube opening } \\
\text {-Measurements to inform how the tubes } \\
\text { are opened, processed, and subsampled } \\
\text { during BC }\end{array}$ & $\begin{array}{l}\text { Non-pristine } \\
\text { environment; } \\
\text { tubes sealed }\end{array}$ & $\begin{array}{l}\text { 1. Describe and remove dust from } \\
\text { the exterior of the tubes } \\
\text { 2. Check tube seals (if possible without } \\
\text { disturbing sample or headspace gas) } \\
\text { 3. Magnetic measurements } \\
\text { 4. Penetrative imaging }\end{array}$ & Section 3 \\
\hline $\begin{array}{l}\text { Basic Characterization }(\mathrm{BC}) \text { : } \\
\text {-Non-invasive measurements } \\
\quad \text { (should be the same for each sample) }\end{array}$ & $\begin{array}{l}\text { Pristine } \\
\text { environment; } \\
\text { tubes in pristine } \\
\text { isolator }\end{array}$ & $\begin{array}{l}\text { 1. Extraction of headspace gas } \\
\text { 2. Opening of tubes } \\
\text { 3. Weighing; photography and imaging } \\
\text { (macro and micro) }\end{array}$ & Section 4 \\
\hline $\begin{array}{l}\text { Preliminary Examination (PE): } \\
\text { - Measurement of selected samples } \\
\text { or subsamples to further develop } \\
\text { the sample catalog and make } \\
\text { effective allocation decisions. } \\
\text { (differs between samples) }\end{array}$ & $\begin{array}{l}\text { Non-pristine } \\
\text { environment; } \\
\text { tubes opened, } \\
\text { sample } \\
\text { removed }\end{array}$ & $\begin{array}{l}\text { 1. Subsampling } \\
\text { 2. Sample preparation } \\
\text { (e.g., polished section; } \\
\text { solvent extraction, etc.) } \\
\text { 3. More detailed measurements/ } \\
\text { analyses }\end{array}$ & Section 5 \\
\hline
\end{tabular}

\footnotetext{
${ }^{\$}$ Appropriate time-sensitive measurements would also be undertaken at each stage.

*Note that non-pristine environment does not imply "not clean." Rather, it implies a slightly less stringent level of cleanliness and a greater number of allowable materials, and it requires contamination controls appropriate for the measurements to be conducted.
}

The subsequent Basic Characterization (BC) steps should be done in a pristine isolator to ensure sample integrity and prevent contamination. A pristine isolator is a clean controlled container (isolator/glovebox/isolation chamber) with a controlled atmosphere where $\mathrm{BC}$ will take place. The main difference between a pristine container and non-pristine container or environment is that the pristine container allows for a highly restrictive list of allowed materials. Once a sample is removed from its pristine container, it would not be allowed back into a pristine container. Both pristine and non-pristine sample containers and environments will have strict contamination control requirements.

To avoid cross contamination between samples and activities, dedicated isolators should be used and cleaned between samples. Tait et al. (2022) proposed that the number of pristine isolators needed for $\mathrm{BC}$ is at least twelve (see Tait et al., 2022, Section 4.2 for full discussion). The number of pristine isolators is expected to have a direct correlation with the necessary size of the facility and the speed at which the sample catalog can be prepared.

To limit contamination while samples are in pristine isolators, techniques will be confined to subsampling with tools of accepted materials with limited sawing capacity. For the PE phase, more extensive sample preparation techniques would be required, for example, production of polished sections. These activities should occur in a controlled environment, though with less stringent requirements than those required for $\mathrm{BC}$. The final contamination control requirements and contamination control and knowledge plan should be developed by a future planning group.

2.2.2. Time-sensitive measurements. Based on the work of Tait et al. (2022), as described in Section 2.2.1 of this report, it is clear that extracting the samples from the returned spacecraft, performing a preliminary examination, completing the SSAP, and producing sample splits for sample allocation is likely to take several months per sample, and perhaps 2 years (or more?) before all of the samples could be made available to be allocated to existing research labs. This raises an important issue: some of the scientific attributes of the Mars samples will degrade with time, beginning at the point that the seals on the sample tubes are penetrated and the samples are no longer in equilibrium with martian headspace gas (Figure 3).

Analysis of these concerns (Tosca et al., 2022) has shown that there are several important investigations where the sample attribute of interest to science is significantly degraded within a time scale of weeks to months. The most important degradation effects are as follows:

(1) Degradation of organic material (including volatile hydrocarbons)

(2) Modification of sample headspace gas composition

(3) Mineral-volatile exchange (including hydrous sulfate minerals, poorly crystalline and X-ray amorphous materials, phyllosilicate minerals, and hydrous carbonate minerals)

(4) Oxidation / reduction of redox-sensitive elements in diverse host phases

MSPG (2019a) and Tosca et al. (2022) strongly recommend that the analytic capabilities needed to carry out timesensitive scientific investigations are placed within the SRF so that those sample measurements can be expedited as part of the early work flow. This is especially important for attributes that degrade on a time scale of weeks to a few months. These are properties that will need to be measured quickly or the opportunity to make such measurements may be irretrievably lost. There are additional investigations that have time sensitivities with a time scale of multiple months to years, though it should be possible to expedite the work flows so those studies can be performed outside the SRF. Conversely, measuring sample attributes that degrade on a time scale of minutes/hours/a few days (some of which have been identified by Tosca et al., 2022) may be difficult to implement. It is important to note that all the investigations that have been determined by Tosca et al. (2022) to be time- 


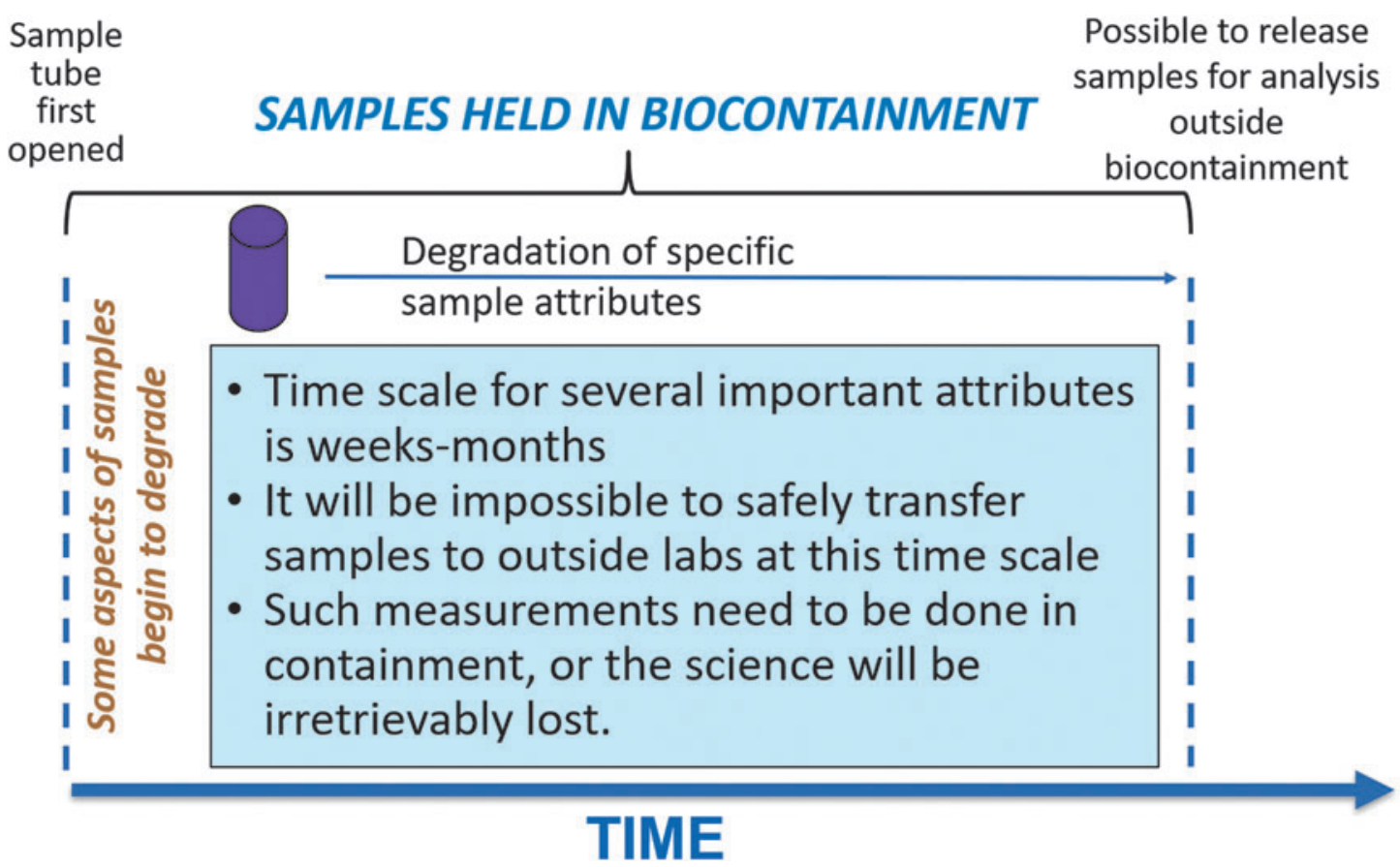

FIG. 3. Schematic illustration showing the importance of being able to carry out certain time-sensitive investigations before the samples can be transferred to labs outside the SRF (modified after MSPG, 2019a; Tosca et al., 2022).

sensitive involve measurement of sample properties that have also been determined to be sterilization-sensitive, and thus quickly sterilizing sample splits and doing these analyses outside the SRF is not an option.

2.2.2.1. Implications for SRF capabilities. In response to the above considerations, Tosca et al. (2022) presented the following four goals for sample analysis inside the SRF:

(1) Characterize sample tube headspace gas composition;

(2) Characterize organic matter of potential biological origin (including volatile hydrocarbons);

(3) Characterize mineral-bound volatiles;

(4) Characterize solid-phase volatile hosts.

Tosca et al. (2022) also produced a traceability matrix that summarizes the goals of time-sensitive activities needed inside the SRF, with corresponding measurements and reference instruments that would be sufficient to carry out those activities. The traceability matrix and the reference instrument types, which have been incorporated into the integrated instrument list, can be viewed in Supplement 2 of this report.

There are several other recommendations for capabilities needed within the SRF to support measurement of timesensitive sample attributes:

(1) The SRF should have the capacity to store subsamples in hermetically sealed containers at sub-zero temperatures if (1) evidence of martian biology or (2) volatile hydrocarbons and/or reactive O-containing species are detected to prevent further degradation of these components, which can occur at ambient temperatures.

(2) Sample tubes should be placed in a secondary container as soon as is feasible. The headspace of this secondary container should be subject to periodic monitoring of gaseous species to ensure that the sample tube seals have not been breached and the samples have not continued to outgas. This should include, for example, headspace composition via optical methods (e.g., tunable laser spectroscopyTLS).

(3) The headspace gas present in a sample tube selected for analysis should be retrieved and characterized compositionally (e.g., using TLS) as soon as is feasible after the seal of the collection container is breached. The retrieval process should recover all available gas originally contained within one individual sample tube volume.

2.2.3. Sterilization-sensitive measurements. Although the probability of finding extant martian life in the MSR samples from the surface of Mars is extremely low (Carrier et al., 2020; National Research Council, 1997; Rummel et al., 2014), one of the guiding principles of MSR will be to keep the samples under BSL-4 (or BSL-4 equivalent) containment until the samples have either been deemed safe for release through completion of the SSAP or through sterilization. For measurements that can be made effectively on sterilized samples, there is no need to consider accommodating those measurements inside the SRF, as sample splits can be sterilized and released from the SRF for analysis relatively quickly. In fact, as discussed by the first MSR Science Planning Group (MSPG, 2019a), it is preferable to plan for as many scientific investigations as possible outside the SRF in specialized laboratories. This course of action is preferable both in terms of limiting the cost of the SRF as well as for scientific reasons, such as access to specialized instruments and the capability to verify the reproducibility of results by using multiple 
instruments or techniques. and having those measurements made in different laboratories. For sample attributes that may be damaged via the sterilization process (i.e., sterilization-sensitive attributes), it would be necessary to plan for analysis of those attributes inside biocontainment or assume that the analyses can be done at a later date after the samples have (presumably) been determined to be nonhazardous (Figure 4).

The first step in determining which investigations might need to take place inside the SRF is to determine those sample attributes that are sterilization-sensitive. MSPG2's Sterilization-Effects Focus Group was asked to evaluate the implications of two sterilization techniques on various sample attributes of interest to MSR science (Velbel et al., 2022).

(1) Sterilization by Dry-Heat:

(a) $180^{\circ} \mathrm{C}$ for 3 hours

(b) $250^{\circ} \mathrm{C}$ for $30 \mathrm{~min}$

(2) Sterilization by exposure to gamma $(\gamma)$ irradiation:

(a) 1 MGy (100 Mrad)

Velbel et al. (2022) divided their analysis into four categories and determined which measurements relevant to each those categories were sterilization-sensitive, with the following conclusions:
(1) Extant or recent martian life: Studies of extant life (either indigenous or contaminants, viable or dead cells) cannot credibly be done on samples that have been sterilized by any means. It is a very high priority that these experiments be done on unsterilized samples inside biocontainment.

(2) Biosignatures of past martian life: Many investigations of potential biosignatures, particularly organic molecular biosignatures, would be severely compromised by either heat or gamma irradiation sterilization techniques. These investigations would need to be done on unsterilized samples inside biocontainment, although there may be alternative sterilization techniques that would allow some of the analyses to be done outside biocontainment.

(3) Geological materials: Sterilization by either heat or gamma irradiation will irreversibly damage samples produced by low temperature surface and nearsurface processes on Mars and render them unsuitable for the required analytical investigations. This includes materials containing volatile-rich minerals and amorphous materials. Hence for investigations of habitability-related (low temperature of formation/ modification, surface/near-surface) geological materials, both dry heat sterilization and gamma irradiation should be avoided.

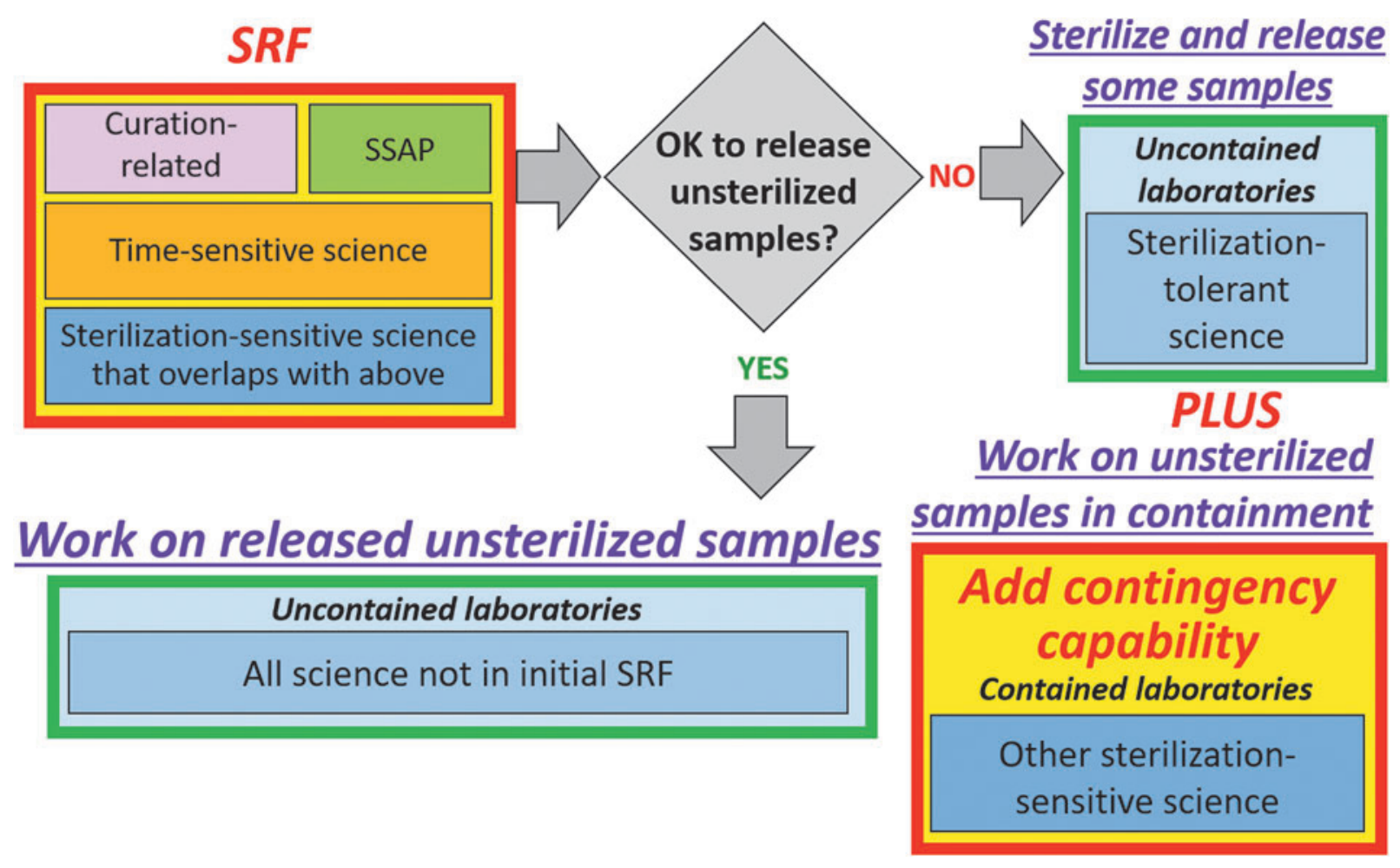

FIG. 4. Flow diagram describing the approach to sterilization-sensitive science. The initial SRF would need to accommodate initial sample characterization, science investigations necessary to complete the SSAP, and time-sensitive science investigations for the reasons described in this report. Once it is determined whether the samples are free of biohazards, two possible scenarios exist. If it is possible to release unsterilized samples ("YES" path in diagram), then all other measurements can be made outside the SRF in uncontained laboratories. If it is not possible to release unsterilized samples ("NO" path in diagram), then most of the remaining measurements can be done on sterilized samples outside biocontainment, but some capability will be needed for additional sterilization-sensitive science to be done inside biocontained laboratories. 
(4) Gas samples: Some gaseous species that science would want to measure in the gas samples may be sensitive to dry heat or gamma irradiation, but the noble gases are expected to be sterilization tolerant. It may be possible to render gas samples sterile via filtration through an inert grid to allow them to be analyzed outside biocontainment.

Potential alternative sterilization techniques identified by Velbel et al. (2022) and noted above might allow some investigations to be conducted outside biocontainment. These include sterilization of liquid extracts by solvent extraction and acid hydrolysis, which could allow organic materials to be characterized outside biocontainment without heat or gamma irradiation sterilization (see Velbel et al., 2022 Section 2.3 for more information). It may also be possible to filter gas samples by means of filtration through an inert mesh (see Velbel et al., 2022, Section 6.4) with nanometer-size openings. Both techniques should be studied further to determine whether these sterilization methods would be acceptable to both science and planetary protection stakeholders.

2.2.3.1. Implications for SRF capabilities. A number of sterilization-sensitive sample attributes have been identified by Velbel et al. (2022), but several of these are neither timesensitive nor necessary for completing the SSAP. Because the intention is to propose requirements for a minimalist SRF, the baseline plan proposed herein includes only the capabilities needed to complete the SSAP, as these will be required to determine whether the samples contain martian biological organisms, as well as to carry out the measurements deemed time-sensitive. Other sterilization-sensitive investigations can be deferred until the samples have been evaluated via the SSAP and, presumably, deemed safe for release (Figure 4). If the results indicate the presence of a potential biohazard or if the results are ambiguous, the SRF should have the capability to allow instruments to be added later (Figure 4). This will be especially important if martian life is discovered; scientists would certainly want to perform a number of investigations related to characterizing and understanding that life, and the public and government sponsors would be equally invested in making the most of such a historic discovery. This does not necessarily mean that the SRF itself would have to expanded or that instruments would have to be brought into the existing building. This could potentially be implemented with a second structure at the same site or even a new or modified building at a different site.

In response to the above considerations, Velbel et al. (2022) presented the following four goals for sample analysis inside the SRF:

(1) Characterize sample tube headspace gas composition;

(2) Detect organic biosignatures of extant and past/extinct life \& characterize organic material of potential biological origin (including volatile hydrocarbons);

(3) Measurements of paleoenvironment and paleohabitability indicators, potential biosignature preservation indicators, and potential physical and inorganic-chemical biosignatures; Characterize solidphase hosts of volatiles;

(4) Characterize mineral- and other solid-phase (amorphous material)-bound volatiles.
The traceability matrix and the reference instrument types relevant to achieving these four goals for sample analysis have been incorporated into the integrated instrument list and can be viewed in Supplement 2 of this report.

Several recommendations for capabilities needed within the SRF to support measurement of sterilization-sensitive sample attributes include the following:

(1) The SRF should have the capability to allow instruments to be added at a later date to allow investigations of other sterilization-sensitive attributes if it is not possible to release unsterilized samples from the SRF. This capability would presumably be needed in any case to replace or repair damaged instruments.

(2) Because all samples are altered to some degree by heat or gamma irradiation sterilization, the heat and gamma-ray sterilization chambers in the SRF should be able to monitor weight loss and the chemical and stable isotopic compositions of carbon, hydrogen, nitrogen, oxygen, and sulfur, and the abundance of phosphorus and other volatiles released during the sterilization step for all samples to characterize and fully document any sterilization-induced alteration.

(3) The SRF should have the capability to implement alternative sterilization techniques such as acid hydrolysis and gas filtration if they can be determined to be acceptable to both science and for planetary protection.

2.2.4. Sample Safety Assessment Protocol. Article IX of the United Nations Outer Space Treaty governs the principles and activities of space exploration to include preventing the "harmful contamination and also adverse changes in the environment of the Earth resulting from the introduction of extraterrestrial matter." Planetary protection policies and implementation standards developed by NASA and ESA must remain consistent with COSPAR guidelines. To that end, the COSPAR Bureau has established a Sample Safety Assessment Protocol Working Group (SSAP-WG) to review and update safety assessments related to transporting, storing, and analyzing unsterilized material from Mars after landing on Earth that had been previously described in the Draft Test Protocol (Rummel et al., 2002). The SSAP-WG includes individuals with relevant expertise in life detection, biohazard analysis, public health, infectious diseases, physical and chemical characteristics of martian surface materials, extraterrestrial sample curation, statistical analysis, and highlevel biological containment. Membership includes international representatives of the science community, members of advisory groups to regulatory agencies, space agencies, and other interested parties. An SRF would need to meet all relevant planetary protection requirements intended to prevent inadvertent contamination of Earth's biosphere, including biosafety containment of all martian material until it has been determined to be non-hazardous or rendered so by sterilization (Pratt and Smith, 2020).

The SSAP will be limited to the assessment of indications of martian life, extant or recently deceased, in any martian material and spacecraft hardware exposed to martian material. The scope of the SSAP is focused on any risk to Earth from "replicating biological entities," a feature consistent with reports from the NRC-SSB and ESF-ESSC reports (National Research Council 2009; Ammann et al., 2012). The evaluation of martian material for potential hazards 
should focus exclusively on searching for evidence of living organisms, their resting states, or their remains (if recently deceased). If evidence of martian life or recent remains are detected, it would be highly unlikely that this life could be determined reliably as either harmful or harmless to Earth, or to prove that all life was in fact deceased, even if it is exhaustively characterized (e.g., by sequencing, cultivation, and biochemical analysis). This is problematic even for terrestrial biological agents, and most of the time limited to very narrow test cases for public health.

Hence the overall scope of the SSAP test sequence, and downstream decision criteria for success, are essential components to understanding the presence of extinct or extant martian life in the returned samples. For the sample safety assessment, each sample tube is considered a separate sample. A negative result in one tube cannot necessarily be applied to all tubes. Information gained in analyzing individual tubes will inform subsequent analysis of other tubes. The elements of the SSAP shall be built on Bayesian analysis, subsampling, a defined test sequence, and peerreviewed decision criteria. Additional information can be found in the SSAP Working Group Final Report (see Kminek et al., 2021).

To mitigate any unnecessary bias introduced during the updated SSAP, there is a need to apply analytical methods that do not presuppose knowledge of the characteristics of an extraterrestrial life form (agnostic approaches) to samples that may have unprecedented chemical complexity. Additionally, leveraging two or more complementary agnostic life detection approaches would also be critical. Each sample tube needs to be scanned by XCT (X-ray Computed Tomography) and correlated chemical mapping early in the process, and dedicated analytical developments are necessary to establish sensitivity and specificity of each science investigation used in the SSAP evaluation and of the integrated sequence.

2.2.4.1. Implications for SRF capabilities. The SRF capabilities associated with the SSAP should include all instrumentation and infrastructure necessary to carry out the test sequence. Based on preliminary information received from the SSAP-WG, MSPG2 has inferred the following necessary capabilities:

(1) Instrumentation to conduct physical sample characterization of the sample at a spatial resolution of 5-20 microns to identify surfaces, veins, and void spaces connected to an exterior surface. This can be done on a sample still inside the tube;

(2) Instrumentation necessary for head gas sampling from sample tubes;

(3) Tools and infrastructure to support sample preparation for solvent extraction using acid hydrolysis. This technique can be used for internal analysis within SRF or, potentially, to sterilize samples for release outside the SRF;

(4) Instrumentation to interrogate sample textures and associated chemistries through high-resolution spatial and spectroscopic analysis of free surfaces and cracks/veins. The spatial resolution for morphological and associated chemical mapping shall be sub-micrometer in size;

(5) Instrumentation for organics analysis by highresolution chemical imaging sample analysis and bulk sample analysis;
(6) Instrumentation to analyze molecular patterns and capture information about isomers and enantiomers in multiple compound classes (e.g., amino acids, lipids, bases, or compounds that could serve analogous biological functions in potential martian organisms);

(7) Instrumentation to identify macromolecules and capture information about polymers (e.g., peptides, nucleic acids, saccharides, or compounds that could serve analogous biological functions in potential martian organisms);

(8) Instrumentation to detect terrestrial life or "life as we know it." This step requires amplification or sub culturing in conditions suitable for propagating terrestrial biology;

(9) Infrastructure to render samples non-hazardous by means of sterilization or other means.

Ultimately, the SSAP will focus exclusively on risk associated with a putative replicating biological entity. Reference instruments needed to accomplish the above test sequence have been proposed by the MSPG2 SRF Focus Group and reviewed by members of the SSAP-WG. These reference instruments should not be assumed to be the final instruments that will be installed in the SRF, but rather serve as a guideline for cost and engineering estimates. These reference instrument types appear in Supplement 2 of this report. The final report from the SSAP will provide input to design considerations for the SRF by further refining the types of preparative processes and analytical instruments needed to search for evidence of extant life in the returned martian samples.

2.2.5. Sample processing, preparation, and storage. To carry out the activities and investigations described above and allow for effective preparation of subsamples for investigations both inside and outside the SRF, it would need to contain a variety of sample processing and preparation capabilities. This includes capabilities needed to divide samples into subsamples as well as manipulate and prepare small particles and larger subsamples with strict contamination control protocols. To prepare samples for various analyses would require an array of laboratory capabilities for sample preparation that include:

(1) Dry wire saws, equipment for powdering, grinding, polishing, and coating samples;

(2) Wet chemistry facilities and equipment to perform solvent extraction and derivatization;

(3) Microbiological laboratory functionalities that include capabilities related to biological sample preparation, refrigerators, and freezers;

(4) Capability to clean and sterilize small tools and equipment that will come in contact with the samples;

(5) Gas extraction and handling capabilities (see Section 2.2.6).

More information on sample prep capabilities can be found in Appendices B, D, and E. The samples would also need to be stored under conditions that protect them from contamination and environmental extremes as described in Section 2.1 and Supplement 1.

2.2.6. Considerations for gas retrieval and analysis. Capabilities related to gas extraction and analysis have been identified by three MSPG2 focus groups, with many 
requiring further work to define the optimal methods for retrieving and analyzing these samples. This process will begin with the need to extract and capture the gas behind each sealed layer of the spacecraft for analysis. It would also be beneficial to determine the pressure inside these sealed areas prior to gas extraction, if possible. At the time of writing there is expected to be only one hermetic seal external to the sample tubes, which is that of the primary containment vessel (PCV) that encapsulates the Orbiting Sample container (OS). The pressure and composition of this gas could be used to determine whether the seal(s) has leaked and to what degree terrestrial atmosphere and potential contamination have been introduced into the OS and potentially into the sample tubes.

Once the gas exterior to the sample tubes has been extracted and the OS has been opened, the next steps would relate to the gas inside each sample tube. Ideally, we would like to be able to measure the leak rate of each sample tube as soon as possible after its removal from the OS. The "best" samples for some science investigations will be those with the lowest leak rates, and this will need to be documented as part of the sample catalog (Tait et al., 2022 Section 3.2.2). If a method to do this could be designed that would not disturb the gas or solid sample inside the tube then this scenario would be favored, but this could very likely be impossible. Traditional methods for measuring leak rates (e.g., He leak rate tests) require accessing both sides of a seal. It may be necessary to wait to test the seal leak rate after gas has been extracted and the sample has been removed from the tube.

It is not advisable to remove the headspace gas from all the tubes prior to the point at which the sample will be extracted, as the sample will begin to undergo alteration once it is removed from its equilibrium with the headspace gas in each tube. This essentially "starts the clock" for many of the timesensitive measurements, so the headspace gas should remain undisturbed until shortly before the solid sample is removed from the tube. Because the gas will not be extracted immediately, each tube should be placed in an individual sample tube isolation container (STIC) that incorporates the capability to allow for periodic extraction (and subsequent analysis) of gases that may be leaking from the sample tube into the secondary container. This would have benefits for both understanding the seal leak rate and for monitoring volatiles that have degassed from the solid samples into the gas phase (Tait et al., Section 3.2.3; Tosca et al., 2022, Section 6.1).

Once a sample tube is selected to be opened, the headspace gas will need to be extracted and transferred, in an unaltered, unfractionated state, to one or several sample vessels that could then be stored or transferred for analysis outside the SRF. These vessels would need to be designed to accommodate potential sterilization of the gas samples by heat or gamma irradiation, including selecting and preparing the containers in such a way as to minimize contamination or alteration of the sample during sterilization. Of particular concern are the potential degassing of terrestrial atmospheric contaminants from the vessel walls and the triggering of chemical reactions between the vessel walls and gaseous molecules. Interactions between vessel walls and gas could be minimized, at least in the case of noble gases, if the containers were baked and degassed sufficiently, for example to a few hundreds of degrees Celsius for a few days prior to use (Velbel et al., 2022 Section 6.2).
The method and apparatus used to extract and transfer the gas into these vessels will require significant further work. The sample tubes do not have a valve or port that can be used for gas extraction, so the tube will have to be penetrated by a yet-to-be-designed apparatus that will collect the headspace gas. There are several important considerations that must be factored into this process including (1) the need to collect ALL of the headspace gas, as this is a very small volume of material, every molecule that can be collected is important for science and any incomplete collection may cause fractionation, (2) this extraction needs to be performed without introducing terrestrial gaseous contamination into the gas samples or the sample tubes, (3) the extraction processes must not introduce contamination into the sample tubes and compromise the pristine nature of the solid samples, which means that the materials used in the extraction system may need to be limited to the materials that are permitted to contact pristine samples. It is highly recommended that a team comprised of engineers, curators, and scientists is formed to address the development of the gas extraction system.

Capabilities for gas sample analysis inside the SRF are primarily driven by the need to understand whether the gas has been subject to terrestrial contamination, and to what degree, and potentially to what degree martian gas has escaped from the sample tubes into the OS. This will inform which gas samples (and related solid samples) are appropriate for scientific analyses based on their relative degree of contamination. Two types of instruments have been recommended for these analyses (Supplement 2, Table C-1, rows 23 and 24). A final decision on the suitability of a specific set of instrumentation to analyze the low volume and molar quantities expected should be made later, as the relevant techniques are rapidly evolving, particularly in the area of optical laser spectroscopy.

It is anticipated that many measurements of noble gas species in the gas samples could be performed on heatsterilized samples outside biocontainment, but this would alter other gas species, and so it would only be appropriate if the subsample were used only for noble gas measurements. Gamma irradiation may also be acceptable for noble gas measurements of subsamples, but this would need to be verified through further experimentation and analyses. Other volatile species are likely to be affected to a greater or lesser extent by either heat or gamma irradiation sterilization processes. There is significant work to be done to better understand the effects of traditional sterilization techniques on these types of samples. Another potential sterilization method for gas samples would be sterilization by filtration. Theoretically, a gas sample could be passed through a grid composed of an inert material with a low outgassing rate and a pore size small enough to effectively remove any particles large enough to be of concern as a potential biohazard. More work needs to be done to determine whether this would be an acceptable alternative, both from the planetary protection and science perspectives. Experiments are needed to investigate possible adsorption/desorption effects that could compromise the samples' composition (desorption of terrestrial gases, adsorption and fractionation of martian gas, kinetics of filtering) (Velbel et al., 2022, Section 6.2).

Please see Section 5 of this report for a list of open questions and areas of future work related to the extraction and analysis of returned martian gas samples. 
2.2.7. Planning for analytical instrumentation in the SRF. 2.2.7.1. Required capabilities and reference instruments. Based on analysis of reports by Kminek et al. (2021), Tait et al. (2022), Tosca et al. (2022), and Velbel et al. (2022), as described above, we conclude that for the SRF to meet its primary objectives, it should have the analytical capabilities needed to carry out the following:

(1) The initial sample characterization (including Pre$\mathrm{BC}, \mathrm{BC}$, and PE), development of the sample catalog, and preparation of the samples for allocation and scientific investigations,

(2) Science investigations necessary to complete the SSAP,

(3) Certain science investigations that are both time- and sterilization-sensitive.

To successfully carry out the above, the SRF would also need to include the capability to:

(a) Maintain strict contamination control and contamination knowledge protocols, including capabilities for monitoring and cleaning large and small equipment and instruments,

(b) Enable multiple sample preparation and processing capabilities,

(c) Accommodate isolators and gloveboxes for sample processing.

The MSPG2 focus groups and SSAP-WG have identified the range of sample properties that would need to be measured inside the SRF to accomplish the objectives noted above, and these have been used to develop the proposed SRF Design Requirements presented in Supplement 1. These requirements are phrased in terms of the capability to measure certain sample properties rather than requirements to provide specific instruments. This is in keeping with our technical approach, described in Section 1.4, to provide capabilitydriven requirements and leave implementation options open for further investigation. It is expected that at least some of the instruments chosen for use inside the SRF will be selected through a future competitive process (Haltigin et al., 2022), and the detailed performance specifications of these instruments (e.g., required spatial resolution, specific wavelengths to be used for optical methods, etc.) will need to be determined by a future planning group such as a Measurement Definition Team (MDT) or an Operations and Requirements Definition Team (ORDT, see MSPG, 2019c).

Although the specific instruments that will be installed in the SRF cannot be determined at this time, we acknowledge that the engineers and architects are beginning accommodation and trade studies related to SRF planning, and these studies would benefit from having more information regarding the estimated number of instruments and approximate information about size, power, thermal, interfaces, and associated sample preparation needs related to those instruments. To aid in this process, we have chosen to present what we are calling "reference instruments" that represent instruments that are currently being used by the science community to make the types of measurements that have been identified by MSPG2 and the SSAP-WG. In doing so, we are specifically not endorsing specific models or manufacturers of these instruments; we are fully supportive of future competitive processes.
To produce the list of reference instruments, each MSPG2 focus group prepared an instrument traceability matrix (Supplement 2) in which their measurement goals are matched with known instrument types that could be used to make those measurements. The lists of instrument types were compiled and evaluated for overlap between the three focus groups and with the instrument list provided by the SSAP-WG, and this was used to generate a summary list of instrument types (Supplement 2, Table C-1). In summary, we propose a list of $\sim 20-30$ instruments, most of which are benchtop-sized instruments. These instruments, and their associated sample preparation needs, would be necessary and sufficient to enable the initial sample characterization, the science investigations necessary for the SSAP, and certain time- and sterilization-sensitive science investigations that cannot be conducted outside the SRF. Reference instruments and their associated sample preparation needs have been compiled in Supplement 3.

FINDING SRF-2: Although most MSR sample investigations would take place outside of the SRF, the SRF needs to include significant laboratory capabilities with advanced instruments and associated sample preparation systems to enable the MSR science objectives to be successfully achieved.

2.2.7.2. Additional considerations for instrumentation planning. Further considerations related to instrumentation planning have to do with timing and contingency planning for managing risks related to scheduling and the inability to acquire information needed to manage and analyze the samples. Instruments can break down and they will require routine maintenance and recalibration, etc. In addition, placing complicated instruments behind the barrier in a BSL-4-grade containment facility would severely restrict access to service technicians and service equipment (e.g., oscilloscopes, He leak detectors). Thus, it may be too simplistic to assume that, for each measurement needed, exactly one instrument is sufficient to generate the data. The probability and consequences of instrument failure and the need for repair, replacement, or instrument redundancy should be carefully considered by a future planning team, and appropriate contingency plans would need to be developed.

Timing is also an important aspect related to the scope of operation of the SRF. At some point, there would need to be stakeholder-set objectives related to how quickly the MSR samples need to be processed through the SRF and made available for scientific investigation outside of biocontainment (potentially including by sterilization). There are several potential operational scenarios that would result in smaller or larger SRF footprints and different timelines for sample processing. For example, a facility with fewer isolators would likely result in a smaller footprint (and lower cost) but would also be constrained to operate at a slower pace. MSPG2 determined that it did not have enough information to make a single recommendation on this topic at this time, as there are many variables to be considered including various possible operational scenarios for the SRF and stakeholder priorities related to budget and schedule. The implications of this trade-off are significant, and they 
need to be studied carefully once there is an indication of the potential budget for the SRF and relative priorities of the MSR-sponsoring agencies are known.

FINDING SRF-3: Preliminary studies of different operational scenarios should be started as soon as possible to enable analysis of the trade-offs between the cost and size of the SRF and the amount of time needed to prepare the samples for allocation and analysis.

It is important to note that the planning described above is for a minimalist facility, based on the contingency that no martian biohazards are present in the samples. In this scenario, it is assumed that unsterilized samples would be able to be transferred to uncontained facilities after they have been determined to be non-hazardous. The instruments in the SRF would be a small subset of all the instruments that would eventually be applied to the samples. However, in the unlikely scenario where martian life or a non-living biohazard $i s$ discovered, at least some unsterilized samples would need to stay in the SRF indefinitely, and a somewhat broader set of instrumentation would need to be brought to bear. If prepared samples for sterilization-sensitive science cannot be released to outside laboratories in an unsterilized state, then the analytical functionalities (instruments) that make these measurements would be needed inside biocontainment. Therefore, the capability to allow for adding additional capabilities at a later date would be needed, whether inside the SRF or in ancillary biocontained laboratories, in order to perform additional sterilization-sensitive measurements that are not part of the current baseline set of analyses. Preliminary planning by Velbel et al. (2022) has identified four instrument types that would form the basis of these investigations (Supplement 2, page S2-4). Additional science investigations and measurements would also be needed to further characterize the putative martian life, if detected, but preliminary discussions on this topic suggest that these investigations and measurements would not require significant additional capabilities above what has already been recommended.

FINDING SRF-4: The ability to add additional analytical capabilities within biocontainment should be preserved to address the contingency scenario in which unsterilized material is not cleared to be analyzed outside of biocontainment. If potential evidence of martian life were to be detected in the samples, for example, it would be a high priority to conduct further investigations related to any putative lifeforms, as well as to enable other sterilization-sensitive science investigations to be conducted in biocontainment.

\section{Other SRF Capabilities}

Beyond the scientific capabilities outlined above, there are myriad building and engineering requirements needed to complete the design of the SRF and thus support the overall science goals of MSR. The specifics of these requirements are outside the expertise of MSPG2 members and are thus incomplete in their identification in the proposed SRF Design Requirements table and in the discussion sections below. The building and engineering requirements needed to complete the design of the SRF should be addressed by the SRF implementation team and subsequent building contractors.

\subsection{Overall biocontainment building engineering and infrastructure}

The SRF is a critical component needed to enable the MSR Campaign to meet the stringent backward planetary protection and contamination control requirements for reliable sample intake, biocontainment, and curation. To date, only planning and trade-off studies have been implemented regarding a notional SRF. Preliminary drivers for requirements are based on the SRF configuration, scientific requirements, and biocontainment level needed to protect Earth's biosphere and cleanliness needed to preserve the science value of the MSR samples. Current draft requirements state that samples returned from Mars would be placed in BSL-4 equivalent containment until they are deemed safe to be released to outside laboratories, either by analysis or by sterilization. BSL-4 laboratories require specialized design requirements that are outlined in the Biosafety in Microbiological and Biomedical Laboratories (BMBL) publication authored by the U.S. Department of Health and Human Services (U.S. Department of Health and Human Services et al., 2020).

BSL-4 laboratories are typically high-containment facilities with a high degree of redundancy built into the design of their complex air handling systems and liquid effluent decontamination and removal systems. They have data storage and processing and electrical power options that could sustain safe operations during a total loss of power, and they have security measures that consist of physical and technical capabilities. These buildings would typically have supporting lower-level laboratories (BSL-3 and BSL-2) that aid in supplemental research functions.

For the SRF, planning activities indicate that the facility would need to accommodate separate rooms for control/ administration, support labs (e.g., BSL-2 or -3), auxiliary space (e.g., outer change, entry anteroom, shower room, inner change room, mechanical), as well as the actual BSL-4 high-containment space (e.g., suit labs and/or ultra-clean isolator labs).

3.1.1. Capabilities requirements. Draft Level 1 requirements for the SRF indicate that it should be engineered to support the facility and infrastructure needs for a high-value scientific research and curation facility (Supplement 1). In addition to the laboratory space, in and out of biocontainment, the SRF would need to host administrative support space, conference rooms, training labs, and classrooms. While there are no standard layouts or design requirements for a high-containment laboratory, there are several requirements for negative and positive pressure regimes and directional airflow to ensure laboratory pathogens remain contained. For the SRF, in addition to the standard BMBL air pressure requirements and HVAC controls, the following draft requirements were noted, including several for uncontained spaces: 
(1) Physical security

(2) IT infrastructure for data sharing and information security internally and externally

(3) Office space for permanent staff, including administrative staff

(4) Office space for guest staff, including visiting scientists, regulatory personnel, public engagement

(5) Biocontainment training laboratories (for suit labs or BSL-4 suit labs or cabinet labs) in non-containment spaces for all personnel working in biocontainment spaces (U.S. Department of Health and Human Services et al., 2020)

(6) Capability to engage observers outside the biocontainment lab both locally (in person) and remotely

(7) Capability to host international investigators

\subsection{Spacecraft receiving and de-integration}

Current planning calls for the samples to be encapsulated within their individual sample tubes; in the engineering terminology, these are referred to as Returnable Sample Tube Assemblies (RSTAs). Each RSTA will have a "plug" with very tight sealing properties, and these seals will have been established using the capping station of M2020. In the current baseline plan, the collective set of sample tubes, along with any associated witness tubes (in engineering terminology, the Witness Tube Assemblies, or WTAs), will then be loaded into a capsule (referred to as the Orbiting Sample container, or OS), which will have a closable lid that is currently planned to be dust-tight, but not air-tight. The OS will be lifted to Mars orbit, where it will be captured by the Earth Return Orbiter (ERO). Within ERO, the OS will be transferred into the Earth Entry System (or EES), and as part of that, two additional seals will be established around the OS: The Primary Containment Vessel (or PCV) and the Secondary Containment Vessel (or SCV). Once everything is configured properly, the EES will be set on an Earth return trajectory and land at an approved landing site on Earth's surface (one site under consideration is the Utah Test and Training Range in Utah, USA).

A ground recovery team will access the landing site as soon as possible after the landing, and current planning is for them to establish an additional seal around the entirety of the returned spacecraft. It is expected that transportation to the SRF will happen in this state. With regard to the SRF, therefore, the final delivery of the spacecraft is presumed to be by truck, which means the SRF will have to have some sort of a loading dock. Planning for how to transition from a loading dock environment to the clean, biocontained interior of the SRF will entail significant complexity.

When the samples arrive at the SRF, they would be surrounded by at least 5 layers of seals (RSTA, OS, PCV, SCV, field seal at Earth landing site), each with different attributes. There would be an interest in knowing the state of each seal as soon as possible. Two additional considerations are important as follows:

(1) The surfaces associated with the innermost seals would be Mars-dirty and Earth-clean, and this aspect will reverse as we get to outer seals. For example, since the EES will land on soil/mud somewhere on Earth, its outside surface will certainly be heavily Earth-contaminated. As these seals are opened, there would be a need to "break the chain of contact," so that the Earth-clean interiors are opened into Earthclean environments within the SRF.

(2) Some of these seals (especially PCV and SCV) would be intimately integrated into the EES. Thus, gaining access to these seals and getting them open requires a de-integration of the entire returned spacecraft. There is also a desire to capture the gas behind all gas-tight seals between the spacecraft exterior and the RSTAs (at the time of writing this is expected to be limited to the seal on the PCV).

Based on the findings of Grady et al. (2022) and extensively commented on by Tait $e t$ al. (2022), the adventitious dust on the interior surfaces within the PCV that have been exposed to the martian environment is considered to be valuable scientifically, and the SRF needs to include the capability to capture and preserve it. This most directly applies to the outside surfaces of the sample tubes that have been cached on the martian surface. Because of their possibly long exposure time, they may have significant quantities of dust. Even though the OS would be open to the martian environment for a much shorter period of time, its interior may also contain significant dust that has been transferred there by the sample tubes. For all surfaces outward of the outer surface of the OS, there would be engineering efforts to reduce or eliminate the dust, so the usefulness of that material to scientific research would be greatly reduced.

\subsection{Planetary Protection: Sterilization}

The SRF needs to have the capability to sterilize subsamples to allow for analyses to be conducted outside the SRF prior to completion of the SSAP and when it is not possible to conduct the analyses with the subsample in a Planetary Protection-compliant sample holder. Two methods currently under consideration are sterilization by dryheat $\left(180^{\circ} \mathrm{C}\right.$ for $3 \mathrm{~h}$ or $250^{\circ} \mathrm{C}$ for $\left.30 \mathrm{~min}\right)$ or by gamma irradiation (1 MGy dosage). It should be noted that it may take up to 2-3 weeks of continuous exposure to a Co-60 source to achieve a $1 \mathrm{MGy}$ dose. Other alternative sterilization methods for certain sample types have been considered, such as gas filtration or acid hydrolysis of sample extracts. In any case, it would be recommended in the future to conduct analogue experiments to assess the impact of the various sterilization methods on sample properties as a way to propose a protocol to those authorities charged with making planetary protection-related decisions.

The SRF should have the capability to sterilize tools or specific pieces of equipment as part of a cleaning cycle to reduce cross-contamination between samples, and also in case the equipment needs to be taken outside of biocontainment. The sterilization protocols would need to be efficient against unknown pathogens, while maintaining the equipment integrity.

In terms of footprint, gamma irradiation should be the only method that requires significant infrastructure to host a source. Other methods are usually achieved with smaller equipment. Gamma irradiation was also found by Velbel et al. (2022) to be a preferred sterilization modality for some sample attributes, allowing some measurements to be done outside biocontainment that would otherwise need to be 
accommodated inside biocontainment if only heat-based sterilization were used. For these reasons, we recommend gamma irradiation be investigated for its potential use in the SRF to reach a consensus early in the SRF design process on its acceptability as a sterilization method for planetary protection.

\subsection{Sample prep/packaging/shipping}

To efficiently transfer subsamples to external facilities and laboratories, the SRF will need to have the capability to prepare and ship subsamples. This would include the capabilities to ship both sterilized (or subsamples otherwise deemed nonhazardous) to uncontained facilities, as well as the capability to transfer samples or subsamples in planetary protection-compliant BSL-4 contained conditions. Biocontained samples may need to be transferred to other biocontained facilities or transferred to a facility in which they could be examined while in their BSL-4 containers (e.g., a synchrotron facility). Areas in which samples are packaged would need to follow the same environmental and contamination requirements as other laboratory spaces inside the SRF to preserve the science value of the sample or subsample to be transferred.

\section{Discussion}

The analysis and findings in this report lead to several significant implications regarding the design of the SRF. Most, or all, of these are consistent with findings from prior studies, and they are listed here for completeness.

Implication \#1: The samples' initial state will be virtually free of terrestrial contamination, and almost everything that is done to them will cause irreversible contamination. A significant amount of SRF infrastructure will be necessary to maintain and monitor appropriate levels of cleanliness, including capability to perform precision cleaning, monitoring of airflow and various types of contamination, production of ultrapure water and solvents, and equipment sterilization. (Section 2.1.2).

Implication \#2: Throughout the facility, there should be a capability to control and monitor temperature and temperature fluctuations as well as the humidity and composition of atmospheric gas. There should also be a capability to maintain an environment without strong magnetic fields $(<0.5 \mathrm{mT})$ in all settings to which the samples are exposed. (Section 2.1.1).

Implication \#3: Although most MSR sample analyses will take place outside the SRF, the objectives listed above in Section 1.3.1 imply that the SRF will need to include a significant laboratory complex with advanced instruments and associated sample preparation systems. This will have implications for room sizing and configuration, as well as required utilities (power, gas lines, vacuum systems, etc.). In addition, many of the reference instruments identified by MSPG2 are currently incompatible with operation inside isolation cabinets; this implies that some aspects of the SRF could need to be operated as a biosafety suit lab.

Implication \#4: The SRF concept described by MSPG2 is meant to minimize the amount of biocontained space needed and maximize the number of measurements that take place outside the SRF. If SSAP related investigations indicate the potential presence of martian life, this could prevent the re- lease of unsterilized material for examination outside of biocontainment. This would necessitate additional analytical capability inside biocontainment to allow for further examination of putative martian lifeforms, which would be a very high priority, and allow other sterilization-sensitive investigations to be carried out. This does not necessarily mean that the initial SRF building must be expanded. This could potentially be implemented with a second building at the same site or a new or modified building at a different site (Section 2.2.3).

Implication \#5: An important aspect related to the design and operation of the SRF is timing. Preliminary studies of different operational scenarios should be started as soon as possible to enable analysis of the trade-offs between the cost and size of the SRF and the amount of time it will take to prepare the samples for allocation and analysis. More advanced studies should be conducted once the budget and priorities for the SRF have been established by NASA and ESA.

The various activities that should be accommodated within the SRF for the MSR science objectives to be achieved is graphically illustrated in Figure 5.

\section{Recommendations for Future Work}

There have been several areas identified that will require refinement either by future planning groups or via further analysis:

- Evaluate potential strategies related to instrument redundancy or the capabilities related to replacing instruments.

- Advance requirements related to environmental and contamination requirements and the monitoring of:

- Allowable humidity,

- Type of allowable gas under which to store samples and acceptable purity levels for same,

- Organic contamination requirement levels,

- Biological contamination requirement levels,

- Inorganic contamination requirement levels,

- Particulate contamination requirement levels.

- Development of a contamination control and knowledge plan.

- More complete definition of what is needed in various sample prep laboratories.

- Further development of approaches, techniques, and requirements related to the extraction, storage, sterilization, and analysis of martian headspace gas samples.

- Elaboration of instrument specifications needed to eventually produce an Announcement of Opportunity (AO) to solicit those instruments or to allow direct procurement of facility provided instruments.

- Evaluation of alternative sterilization techniques such as solvent extraction/acid hydrolysis or filtration- conduct analogue experiments to assess the impact of the various sterilization methods on sample properties, and as a way to propose a protocol to authorities competent on Planetary Protection.

- Investigation of potential strategies for storing and analyzing soil collected from area surrounding the EES landing site.

- Timing is an important aspect related to the design and operation of the SRF. We recommend conducting an evaluation of the trade-offs between the cost and size of the SRF and the amount of time it will take to prepare 


\section{SRF Concept of Operations}

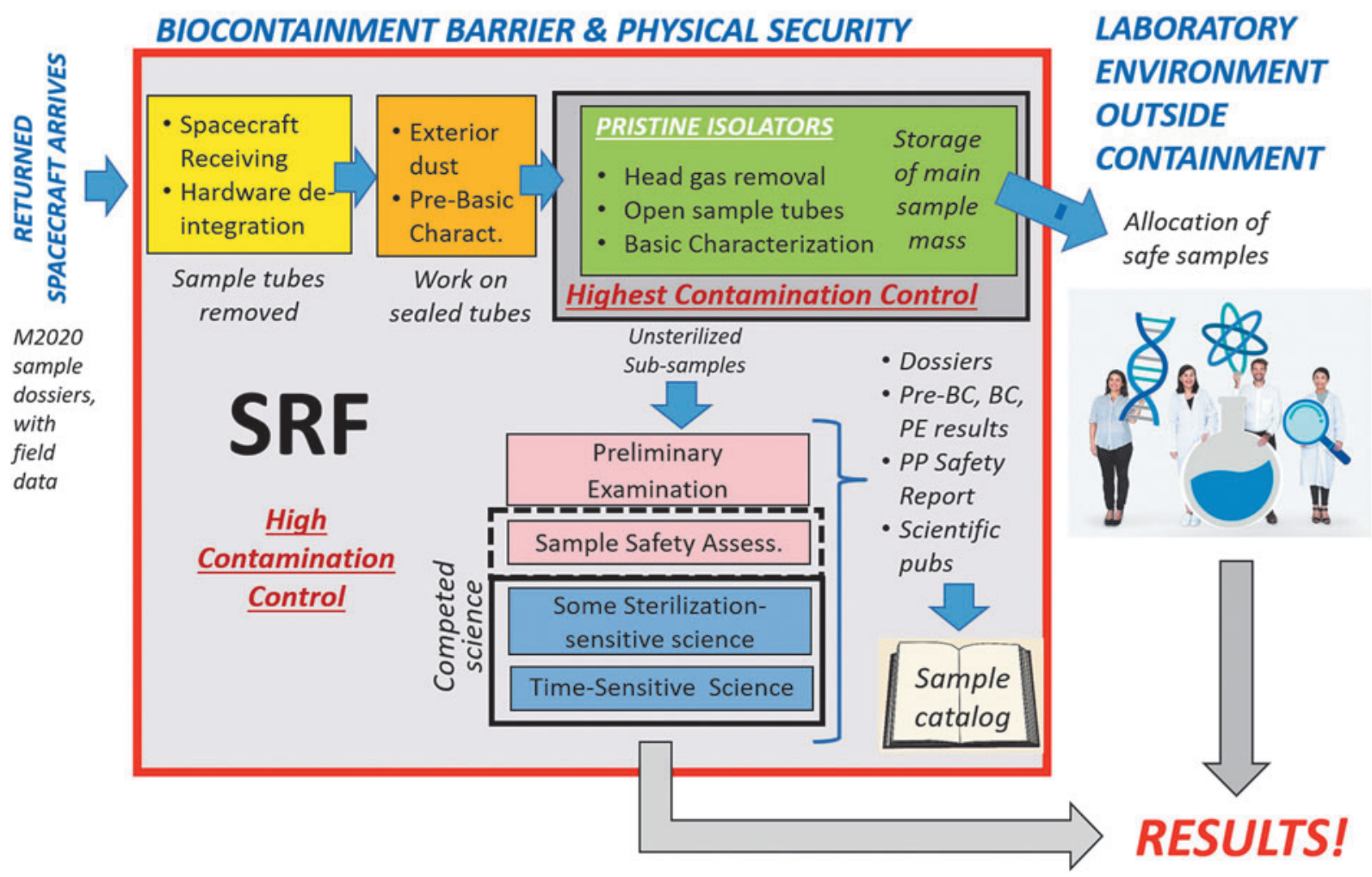

FIG. 5. Summary SRF Workflow Diagram. Note that everything represented inside the red border should be accommodated inside BSL-4 equivalent containment, with varying standards for contamination control. The most stringent contamination control requirements and materials limitations will be needed while the samples are inside pristine isolators. Requirements are expected to be slightly less stringent in the laboratories where PE and other investigations take place and least stringent when accessing the sealed sample tubes from the returned spacecraft.

the samples for allocation and analysis. A smaller facility with fewer isolators for parallel processing than recommended by Tait et al. (2022) (i.e., Section 2.2.1) would likely result in a smaller footprint for the SRF, as well as a slower pace for the initial sample characterization, production of the sample catalog, completion of the SSAP, and other analyses, as well as a delay in sample allocation to external laboratories. This could also impact the time-sensitive science investigations that could be successfully conducted within the SRF. This would presumably also result in a higher lifetime operating cost for the SRF. We recommend that these trades be studied carefully once there is an indication of the potential budget for the SRF and relative priorities of the agencies (i.e., NASA and ESA) are known.

- Evaluate the possibility of using robotics and remote manipulation systems both inside and outside isolators. Further work should be done to evaluate the possible uses of robotics and remote manipulator systems, and the benefits of these systems to reduce contaminations risks related to pressure systems and suit labs.

- Address open questions related to gas extraction and analysis, including:
Is it possible to test the RSTA seal leak rate without extracting the headspace gas first?

- If so, this would have the benefit of not "starting the clock" on the time-sensitive processes that begin to alter the sample once it is no longer in equilibrium with its headspace gas

- How to extract the headspace gas from each sample tube while:

- retrieving all of the gas present in the tube?

- not contaminating the solid samples, keeping them as pristine as possible?

- Need to know the allowable materials

- not contaminating the gas?

- The CC requirements for gas samples still need to be determined.

- not fractionating the gas (i.e., preserving its isotopic composition)?

- Will it be acceptable (for backward PP) to filter gas through nm scale mesh and analyze outside biocontainment?

- Will it be acceptable (in terms of sample quality) to filter gas through nm scale mesh and analyze outside biocontainment? 
- If yes to both of the above, the only analytical capabilities needed inside the SRF are those sufficient to determine the level of terrestrial contamination.

- If no to either of the above, greater (and difficult to implement) capabilities may be needed in the SRF

- Additional work would be needed to fully determine what measurements are sensitive to heat or gamma irradiation sterilization

\section{Acknowledgments}

The decision to implement Mars Sample Return will not be finalized until NASA's completion of the National Environmental Policy Act (NEPA) process. This document is being made available for planning and information purposes only.

\section{Disclosure Statement}

No competing interests.

\section{Funding Information}

A portion of this work was funded by the National Aeronautics and Space Administration (NASA) and the European Space Agency (ESA).

A portion of this work was carried out at the Jet Propulsion Laboratory, California Institute of Technology, under a contract with the National Aeronautics and Space Administration (80NM0018D0004).

This work has partly (H. B.) been carried out within the framework of the NCCR PlanetS supported by the Swiss National Science Foundation. M.A.V.'s participation in MSPG2 was supported in part by a sabbatical leave-ofabsence from Michigan State University. M.-P.Z. was supported by projects PID2019-104205GB-C21 of Ministry of Science and Innovation and MDM-2017-0737 Unidad de Excelencia 'Maria de Maeztu'- Centro de Astrobiología (CSIC-INTA) (Spain).

\section{References}

Beaty DW, Grady MM and iMARS Working Group (2008) Preliminary Planning for an International Mars Sample Return Mission: Report of the International Mars Architecture for the Return of Samples (iMARS) Working Group. White paper available online at https://mepag.jpl.nasa.gov/reports/ iMARS_FinalReport.pdf.

Beaty DW, Allen CC, Bass DS, et al. (2009) Planning considerations for a Mars sample receiving facility: Summary and interpretation of three design studies. Astrobiology 9:745758.

Beaty DW, Liu Y, Des Marais DJ, et al. (2014) Mars returned sample science: Scientific planning related to sample quality [abstract 1208]. In Eighth International Conference on Mars, Pasadena.

Beaty DW, Grady MM, McSween HY, et al. (2019) The potential science and engineering value of samples delivered to Earth by Mars sample return: International MSR Objectives and Samples Team (iMOST). Meteorit Planet Sci 54:S3S152.

Boeder PA and Soares CE (2020) Mars 2020: mission, science objectives and build [abstract 1148903]. In SPIE-Intl Soc Optical Eng, virtual.
Carr MH, McCleese DJ, Bada JL, et al. (1999) Mars Sample Handling and Requirements Panel (MSHARP) Final Report. NASA Technical Memorandum Document ID 19990047590.

Carrier BL, Beaty DW, Hecht MH, et al. (2017) Planning for the scientific use of samples of martian granular materials potentially to be returned by Mars Sample Return [abstract 6292]. In $80^{\text {th }}$ Annual Meeting of the Meteoritical Society, Santa Fe.

Carrier BL, Beaty DW, Meyer MA, et al. (2020) Mars extant life: What's next? Conference Report. Astrobiology 20:785814.

Grady MM, Summons RE, Swindle T, et al. (2022) The scientific importance of returning airfall dust as part of Mars Sample Return (MSR). Astrobiology 22(S1):S-176-S-185.

Haltigin T, Lange C, Mugnuolo R, et al. (2018) iMARS phase 2: A draft mission architecture and science management plan for the return of samples from Mars. Astrobiology 18:S1S131.

Haltigin T, Hauber E, Kminek G, et al. (2022) Rationale and proposed design for a Mars Sample Return (MSR) science program. Astrobiology 22(S1):S-27-S-56.

Liu Y, Beaty DW and Farley K (2014) Mars Returned Sample science: Planning considerations related to the inorganic contamination of geological samples [abstract 248635]. 2014 GSA Annual Meeting. Available online at https://gsa.confex .com/gsa/2014AM/webprogram/Paper248635.html.

Mattingly RL, Smith II AL, Calaway MJ and Harrington AD (2020) Tours of High-Containment and Pristine Facilities in Support of Mars Sample Return (MSR) Sample Receiving Facility (SRF) Definition Studies. White paper available online at https://trs.jpl.nasa.gov/handle/2014/50446.

McLennan SM, Sephton MA, Allen C, et al. (2012) Planning for Mars Returned Sample science: Final report of the MSR End-to-End International Science Analysis Group (E2EiSAG). Astrobiology 12:175-230.

Meyer MA, Kminek G, Beaty DW, et al. (2022) Final report of the MSR Science Planning Group 2 (MSPG2). Astrobiology 22(S1):S-5-S-26.

MSPG (MSR Science Planning Group) (2019a) The Relationship of MSR Science and Containment. Unpublished workshop report available online at https://mepag.jpl.nasa.gov/ reports/Science_in_Containment_Report.pdf.

MSPG (MSR Science Planning Group) (2019b) Science-Driven Contamination Control Issues Associated with the Receiving and Initial Processing of the MSR Samples. Unpublished workshop report available online at https://mepag.jpl.nasa.gov/ reports/MSPG_Contamination_Control_Report_Final.pdf.

National Research Council (1997) Mars Sample Return: Issues and Recommendations. National Academies Press, Washington, D.C.

National Research Council (2002) The Quarantine and Certification of Martian Samples. National Academies Press, Washington, D.C.

National Research Council (2011) Visions \& Voyages for Planetary Science in the Decade 2013-2022. National Academies Press, Washington, D.C.

Pratt LM and Smith AL (2020) Expectations for backward planetary protection planning during Mars Sample Return planning. In 2020 IEEE Aerospace Conference, Big Sky.

Rummel JD, Beaty DW, Jones MA, et al. (2014) A new analysis of Mars "Special Regions": Findings of the second MEPAG Special Regions Science Analysis Group (SRSAG2). Astrobiology 14:887-968. 
Rummel JD, Race MS, DeVincenzi DL, et al. (2002) A Draft Test Protocol for Detecting Possible Biohazards in Martian Samples Returned to Earth. Washington, DC, pp NASA/CP2002-211842.

Tait KT, McCubbin FM, Smith CL, et al. (2022) Preliminary planning for Mars Sample Return (MSR) curation activities in a Sample Receiving Facility (SRF). Astrobiology 22(S1): S-57-S-80.

Tosca NJ, Agee CB, Cockell CS, et al. (2022) Time-sensitive aspects of Mars Sample Return (MSR) science. Astrobiology 22(S1):S-81-S-111.

U.S. Department of Health and Human Services, Centers for Disease Control, and National Institutes of Health (2020) Biosafety in Microbiological and Biomedical Laboratories (BMBL) 6th Edition (PJ Meechan and J Potts (eds.) $\mid$ CDC Laboratory Portal $\mid$ CDC. U.S. Government Printing Office, Washington, D.C.

Velbel MA, Cockell CS, Glavin DP, et al. (2022) Planning implications related to sterilization-sensitive science investigations associated with Mars Sample Return (MSR). Astrobiology 22(S1):S-112-S-164.

For further information about MSPG2, please contact Michael Meyer (Michael.a.meyer@nasa.gov), Gerhard Kminek (Gerhard.kminek@esa.int), David Beaty (dwbeaty@jpl.nasa.gov), or Brandi Carrier (bcarrier@jpl.nasa.gov).

For further information on the technical content of this report, contact Brandi Carrier (bcarrier@jpl.nasa.gov).

Acronyms Used
$\mathrm{AO}=$ Announcement of Opportunity
$\mathrm{BC}=$ Basic Characterization
$\mathrm{BMBL}=$ Biosafety in Microbiological \& Biomedical
$\quad$ Laboratories
$\mathrm{BSL}-4=$ Biosafety Level 4
$\mathrm{CERN}=$ The European Organization for Nuclear
Research

COSPAR $=$ Committee on Space Research

E2E-iSAG $=$ MSR End-to-End International Science Analysis Group

$\mathrm{EES}=$ Earth Entry System

$\mathrm{ERO}=$ Earth Return Orbiter

$\mathrm{ESF}=$ European Science Foundation

$\mathrm{ESSC}=$ European Space Sciences Committee

FT-IR $=$ Fourier Transform Infrared Spectroscopy

iMARS = international Mars Architecture for the Return of Samples

$\mathrm{IMEWG}=$ International Mars Exploration Working Group

iMOST = international MSR Objectives \& Samples Team

MGy $=$ Megagray

Mrad $=$ Megarad

MRSH $=$ Mars Returned Sample Handling

$\mathrm{MSPG}=$ MSR Science Planning Group

MSPG2 = MSR Science Planning Group Phase 2

MSR $=$ Mars Sample Return

$\mathrm{NRC}=$ National Research Council

$\mathrm{OS}=$ Orbiting Sample Container

$\mathrm{PCV}=$ Primary Containment Vessel

$\mathrm{PE}=$ Preliminary Examination

$\mathrm{PI}=$ Principle Investigator

$\mathrm{PP}=$ Planetary Protection

Pre-BC $=$ Pre-Basic Characterization

RSSB $=$ Returned Sample Science Board

RSTA $=$ Returnable Sample Tube Assembly

SCV $=$ Secondary Containment Vessel

$\mathrm{SEM}=$ Scanning Electron Microscope

$\mathrm{SRF}=$ Sample Receiving Facility

SSAP $=$ Sample Safety Assessment Protocol

$\mathrm{SSAP}-\mathrm{WG}=$ Sample Safety Assessment Protocol Working Group

$\mathrm{SSB}=$ Space Studies Board

STIC $=$ Sample Tube Isolation Chamber

STSI $=$ Space Telescope Science Institute

$\mathrm{ToR}=$ Terms of Reference

$\mathrm{WTA}=$ Witness Tube Assembly 